\title{
The Total Synthesis of Coleophomones B, C, and D
}

\author{
K. C. Nicolaou, ${ }^{*}$ Tamsyn Montagnon, ${ }^{\dagger}$ Georgios Vassilikogiannakis, ${ }^{\dagger}$ Casey J. N. Mathison.
}

Contribution from the Department of Chemistry and The Skaggs Institute for Chemical Biology, The Scripps Research Institute, 10550 North Torrey Pines Road, La Jolla, California 92037, and Department of Chemistry and Biochemistry, University of California, San Diego, 9500 Gilman

Drive, La Jolla, California 92093.

Corresponding author e-mail: kcn@scripps.edu

\section{Supporting Information}

\section{Experimental Data for Compounds}

General Procedures. All reactions were carried out under an argon atmosphere with dry solvents under anhydrous conditions, unless otherwise noted. Dry tetrahydrofuran (THF), toluene, benzene, diethyl ether $\left(\mathrm{Et}_{2} \mathrm{O}\right)$, ethylene glycol dimethyl ether (DME), and methylene chloride $\left(\mathrm{CH}_{2} \mathrm{Cl}_{2}\right)$ were obtained by passing commercially available pre-dried, oxygen-free formulations through activated alumina columns. Yields refer to chromatographically and spectroscopically ( ${ }^{1} \mathrm{H}$ NMR) homogeneous materials, unless otherwise stated. Reagents were purchased at the highest commercial quality and used without further purification, unless otherwise stated. Reactions were monitored by thin-layer chromatography (TLC) carried out on $0.25 \mathrm{~mm}$ E. Merck silica gel plates (60F-254) using UV light as visualizing agent and an ethanolic solution of phosphomolybdic acid and cerium sulfate, and heat as developing agents. E. 
Merck silica gel (60, particle size $0.040-0.063 \mathrm{~mm}$ ) was used for flash column chromatography. Preparative thin-layer chromatography (PTLC) separations were carried out on 0.25 or $0.50 \mathrm{~mm}$ E. Merck silica gel plates (60F-254). NMR spectra were recorded on Bruker DRX-600, DRX500, AMX-500 or AMX-400 instruments and calibrated using residual undeuterated solvent as an internal reference. The following abbreviations were used to explain the multiplicities: $\mathrm{s}=$ singlet, $\mathrm{d}=$ doublet, $\mathrm{t}=$ triplet, $\mathrm{q}=$ quartet, $\mathrm{m}=$ multiplet, quin $=$ quintuplet, sext $=$ sextet, $\operatorname{sep}=$ septet, $\mathrm{br}=$ broad, $\mathrm{AB}=\mathrm{AB}$ quartet, $\mathrm{app}=$ apparent. IR spectra were recorded on a Perkin-Elmer 1600 series FT-IR spectrometer. Electrospray ionization (ESI) mass spectrometry (MS) experiments were performed on an API 100 Perkin Elmer SCIEX single quadrupole mass spectrometer at 4000V emitter voltage. High-resolution mass spectra (HRMS) were recorded on a VG ZAB-ZSE mass spectrometer using MALDI (matrix-assisted laser-desorption ionization).

\section{Monoprenylated vinylogous ester 6b (Scheme 2):}

A solution of 5-methyl-1,3-cyclohexadione $(5.0 \mathrm{~g}, 39.68 \mathrm{mmol})$ in $\mathrm{MeOH}(50 \mathrm{~mL})$ containing $\mathrm{H}_{2} \mathrm{SO}_{4}(98 \%, 0.5 \mathrm{~mL})$ was heated to reflux for $12 \mathrm{~h}$. The solution was then cooled to room

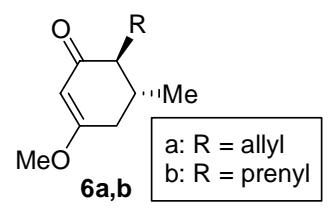
temperature and neutralized ( $\mathrm{KOH}$ pellets). The volume was reduced in vacuo to approximately $10 \mathrm{~mL}$. The residual liquid was diluted with $\mathrm{Et}_{2} \mathrm{O}$ $(50 \mathrm{~mL})$. The organic solution was then washed with brine and dried (anhydrous $\mathrm{K}_{2} \mathrm{CO}_{3}$ ). The solvent was then removed in vacuo to afford the corresponding vinylogous ester $(4.72 \mathrm{~g}, 85 \%)$ as a pale yellow syrup.

$R_{\mathrm{f}}=0.27$ (silica gel, hexane/EtOAc 2:1); IR (film) $v_{\max }=2964,2955,1648,1607,1458,1440$, 1381, 1224, 1140, 1008, 886, 858, $606 \mathrm{~cm}^{-1} ;{ }^{1} \mathrm{H}$ NMR $\left(500 \mathrm{MHz}, \mathrm{CDCl}_{3}\right): \delta=5.30(\mathrm{~s}, 1 \mathrm{H})$, $3.64(\mathrm{~s}, 3 \mathrm{H}), 2.37$ (m, $2 \mathrm{H}), 2.16(\mathrm{~m}, 1 \mathrm{H}), 2.10(\mathrm{dd}, J=16.8,10.3 \mathrm{~Hz}, 1 \mathrm{H}), 1.98$ (dd, $J=16.5$, $11.3 \mathrm{~Hz}, 1 \mathrm{H}), 1.02(\mathrm{~d}, J=6.6 \mathrm{~Hz}, 3 \mathrm{H}) \mathrm{ppm} ;{ }^{13} \mathrm{C} \mathrm{NMR}\left(125 \mathrm{MHz}, \mathrm{CDCl}_{3}\right): \delta=199.5,178.0$, 101.8, 55.5, 44.9, 36.8, 28.7, 20.7 ppm; HRMS (MALDI): calcd for $\mathrm{C}_{8} \mathrm{H}_{12} \mathrm{O}_{2}[\mathrm{MH}]^{+}$: 141.0910, found: 141.0912 .

A solution of the vinylogous ester prepared above $(4.72 \mathrm{~g}, 33.73 \mathrm{mmol})$ in anhydrous THF (150 $\mathrm{mL}$ ) was added slowly to a solution of LiHMDS (35.41 mL, $1 \mathrm{M}$ in THF) in anhydrous THF $(100 \mathrm{~mL})$ at $-78^{\circ} \mathrm{C}$. The solution was then stirred for $1 \mathrm{~h}$ at the same temperature. A solution of 
prenyl bromide $(4.28 \mathrm{~mL}, 37.10 \mathrm{mmol})$ in anhydrous THF $(10 \mathrm{~mL})$ was added, also at $-78{ }^{\circ} \mathrm{C}$, and the resulting solution warmed over a period of $3 \mathrm{~h}$ to $0{ }^{\circ} \mathrm{C}$. The reaction was quenched with saturated aq. $\mathrm{NH}_{4} \mathrm{Cl}(75 \mathrm{~mL})$ and diluted with $\mathrm{Et}_{2} \mathrm{O}(150 \mathrm{~mL})$. The layers were separated and the organic layer phase was washed with brine $(100 \mathrm{~mL})$ and dried $\left(\mathrm{MgSO}_{4}\right)$. The solvent was removed in vacuo and the residue was purified by flash column chromatography (silica gel, EtOAc:hexane $1: 3 \rightarrow 1: 2)$ to afford $\mathbf{6 b}(5.96 \mathrm{~g}, 85 \%)$ as a pale yellow syrup.

The product is a mixture of trans:cis (4:1) diastereoisomers, which could be separated by very careful flash column chromatography. A similar procedure was applied in order to prepare 6a (80\% yield) using allyl bromide as the alkylating agent rather than prenyl bromide.

6b (major diastereoisomer): $R_{\mathrm{f}}=0.53$ (silica gel, hexane/EtOAc 2:1); IR (film) $v_{\max }=2963$, 2913, 1650, 1613, 1442, 1381, 1222, 1160, 1008, $834 \mathrm{~cm}^{-1} ;{ }^{1} \mathrm{H}$ NMR $\left(500 \mathrm{MHz}, \mathrm{CDCl}_{3}\right): \delta=$ $5.31(\mathrm{~s}, 1 \mathrm{H}), 5.01(\mathrm{tt}, J=6.9,1.3 \mathrm{~Hz}, 1 \mathrm{H}), 3.65(\mathrm{~s}, 3 \mathrm{H}), 2.46(\mathrm{~m}, 2 \mathrm{H}), 2.27(\mathrm{~m}, 1 \mathrm{H}), 2.10(\mathrm{~m}$, $2 \mathrm{H}), 2.00(\mathrm{~m}, 1 \mathrm{H}), 1.65$ (brs, $3 \mathrm{H}), 1.61$ (brs, $3 \mathrm{H}), 1.03(\mathrm{~d}, J=6.6 \mathrm{~Hz}, 3 \mathrm{H}) \mathrm{ppm} ;{ }^{13} \mathrm{C} \mathrm{NMR}$ $\left(125 \mathrm{MHz}, \mathrm{CDCl}_{3}\right): \delta=201.1,176.0,132.9,121.3,101.4,55.5,52.3,35.0,30.6,26.7,25.8,19.7$, 17.8 ppm; HRMS (MALDI): calcd for $\mathrm{C}_{13} \mathrm{H}_{20} \mathrm{O}_{2}[\mathrm{MH}]^{+}:$209.1536, found: 209.1536.

\section{Bisprenylated-1,3-cyclohexadione 8b (Scheme 2):}

To solution of LDA at $-78{ }^{\circ} \mathrm{C}$, prepared by treating a solution of diisopropylamine $(3.12 \mathrm{~mL}$, $23.79 \mathrm{mmol})$ in anhydrous THF $(15 \mathrm{~mL})$ with $n$-BuLi $(9.90 \mathrm{~mL}, 1.6 \mathrm{M}$ in hexane) for $20 \mathrm{~min}$,

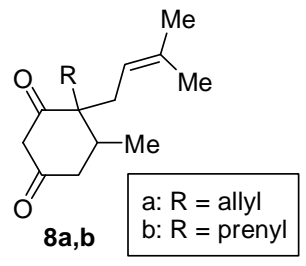
was added slowly (over $30 \mathrm{~min})$ a solution of $6 \mathbf{b}(3.0 \mathrm{~g}, 14.42 \mathrm{mmol})$ in anhydrous THF:HMPA $(7: 1,18 \mathrm{~mL})$. The resulting solution was stirred for $30 \mathrm{~min}$ at the same temperature. A solution of prenyl bromide $(3.33 \mathrm{~mL}$, $28.84 \mathrm{mmol})$ in anhydrous THF $(5 \mathrm{~mL})$ was then added and the temperature was slowly raised to $20^{\circ} \mathrm{C}$ over a period of $12 \mathrm{~h}$. The reaction was quenched with saturated aq. $\mathrm{NH}_{4} \mathrm{Cl}(20 \mathrm{~mL})$ and diluted with $\mathrm{Et}_{2} \mathrm{O}(40 \mathrm{~mL})$. The layers were separated and the organic phase was washed with brine $(40 \mathrm{~mL})$ and dried $\left(\mathrm{MgSO}_{4}\right)$. The solvent was removed in vacuo and the crude residue was purified by flash column chromatography (silica gel, EtOAc:hexane 1:5) to furnish the desired bisprenylated vinylogous ester $(3.54 \mathrm{~g}, 89 \%)$ as a pale yellow syrup.

$R_{\mathrm{f}}=0.67$ (silica gel, hexane/EtOAc 2:1); IR (film) $v_{\max }=2966,2912,1650,1614,1442,1383$, 1221, 1172, 1007, $836 \mathrm{~cm}^{-1} ;{ }^{1} \mathrm{H}$ NMR (600 MHz, $\left.\mathrm{CDCl}_{3}\right): \delta=5.29$ (s, $\left.1 \mathrm{H}\right), 5.04$ (tt, $J=6.2,1.3$ $\mathrm{Hz}, 1 \mathrm{H}), 4.89$ (tt, $J=5.7,1.3 \mathrm{~Hz}, 1 \mathrm{H}), 3.64$ (s, $3 \mathrm{H}), 2.52$ (dd, $J=14.4,5.2 \mathrm{~Hz}, 1 \mathrm{H}), 2.40$ (m, 1 
H), 2.26 (m, $3 \mathrm{H}), 2.06$ (m, $2 \mathrm{H}), 1.65$ (brs, $3 \mathrm{H}), 1.62$ (brs, $3 \mathrm{H}), 1.57$ (brs, $3 \mathrm{H}$ ), 1.56 (brs, $3 \mathrm{H}$ ), $0.96(\mathrm{~d}, J=6.5 \mathrm{~Hz}, 3 \mathrm{H}) \mathrm{ppm} ;{ }^{13} \mathrm{C} \mathrm{NMR}\left(150 \mathrm{MHz}, \mathrm{CDCl}_{3}\right): \delta=202.9,175.1,133.2,132.8$, 120.4, 119.3, 101.6, 55.4, 50.9, 34.1, 33.0, 31.6, 29.5, 26.0, 25.9, 17.8 (2 C), 15.4 ppm; HRMS (MALDI): calcd for $\mathrm{C}_{18} \mathrm{H}_{28} \mathrm{O}_{2}[\mathrm{MH}]^{+}:$277.2162, found: 277.2158 .

The same yield (89\%) was obtained for the vinylogous ester synthesized by prenylation of $\mathbf{6 a}$ using an identical procedure. The same protocol was also applied to the alkylation of $\mathbf{6} \mathbf{b}$ with allylic bromide 20 (Scheme 4) and allylic bromide 34 (Scheme 6).

A solution of the bisprenylated vinylogous ester $(3.54 \mathrm{~g}, 12.83 \mathrm{mmol})$, prepared above, in THF $(100 \mathrm{~mL})$ was treated with $\mathrm{HCl}(10 \mathrm{~mL}, 1 \mathrm{M})$ at $25^{\circ} \mathrm{C}$ for $14 \mathrm{~h}$. The reaction mixture was diluted with EtOAc/ $\mathrm{H}_{2} \mathrm{O}(2: 1,100 \mathrm{~mL})$. The layers were separated and the organic phase was washed with brine $(75 \mathrm{~mL})$ and dried $\left(\mathrm{MgSO}_{4}\right)$. The solvent was removed in vacuo to afford $\mathbf{8 b}(3.19 \mathrm{~g}$, $95 \%$ ) as a pale yellow syrup. The keto- and enol-tautomers of $\mathbf{8 b}$ can both be seen in the NMR spectra (keto:enol, 4:1). Identical procedures were used for the preparation of 8a from its corresponding vinylogous ester and for the hydrolysis of monoaklylated vinylogous esters $\mathbf{6 a}$ and $\mathbf{b}$ to afford the corresponding monoalkylated 1,3-cyclohexadiones 7a and $\mathbf{b}$, respectively.

8b: $R_{\mathrm{f}}=0.21$ (silica gel, hexane/EtOAc 2:1); IR (film) $v_{\max }=2968,2913,1606,1560,1443$, 1407, 1382, 1306, 1227, 1194, $846 \mathrm{~cm}^{-1} ;{ }^{1} \mathrm{H}$ NMR (500 MHz, $\mathrm{CDCl}_{3}$, keto form): $\delta=5.00(\mathrm{tt}, J$ $=7.3,1.5 \mathrm{~Hz}, 1 \mathrm{H}), 4.89$ (tt, $J=7.1,1.3 \mathrm{~Hz}, 1 \mathrm{H}), 3.46(\mathrm{dd}, J=17.2,1.1 \mathrm{~Hz}, 1 \mathrm{H}), 3.29(\mathrm{dd}, J=$ 17.2, $1.4 \mathrm{~Hz}, 1 \mathrm{H}), 2.81$ (dd, $J=16.7,5.5 \mathrm{~Hz}, 1 \mathrm{H}), 2.47$ (m, $2 \mathrm{H}), 2.40-2.20(\mathrm{~m}, 4 \mathrm{H}), 1.68$ (d, $J=1.1 \mathrm{~Hz}, 3 \mathrm{H}), 1.66(\mathrm{~d}, J=1.1 \mathrm{~Hz}, 3 \mathrm{H}), 1.59$ (brs, $6 \mathrm{H}), 0.92(\mathrm{~d}, J=7.0 \mathrm{~Hz}, 3 \mathrm{H}) \mathrm{ppm} ;{ }^{13} \mathrm{C}$ NMR (125 MHz, $\mathrm{CDCl}_{3}$, keto form): $\delta=207.4,204.4,134.9,134.8,118.4,118.1,56.9,54.7$, 44.8, 32.4, 31.5, 29.1, 26.1, 26.0, 17.9 (2 C), 15.8 ppm; HRMS (MALDI): calcd for $\mathrm{C}_{17} \mathrm{H}_{26} \mathrm{O}_{2}$ $[\mathrm{MH}]^{+}: 263.2005$, found: 263.2008

The four-step procedure described above for the transformation of $\mathbf{5}$ into $\mathbf{8 a}$ and $\mathbf{b}$, was also applied to the preparation of 65a and $\mathbf{b}$, starting from 64 (Scheme 13).

\section{Phenolic aldehyde 32 (Scheme 6):}

To a solution of benzylic alcohol $\mathbf{3 1}{ }^{24}(5.0 \mathrm{~g}, 25.77 \mathrm{mmol})$ in $\mathrm{CH}_{2} \mathrm{Cl}_{2}(150 \mathrm{~mL})$ was added $\mathrm{Et}_{3} \mathrm{~N}$ (5.38 mL, $38.65 \mathrm{mmol})$, 4-DMAP (157 mg, $1.28 \mathrm{mmol}$ ), and 4-bromo benzoyl chloride (5.94 g, $27.05 \mathrm{mmol}$ ). The resulting solution was stirred for $20 \mathrm{~min}$ at $25^{\circ} \mathrm{C}$. The organic solution was 


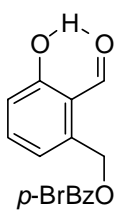

washed with saturated aq. $\mathrm{NaHCO}_{3}(100 \mathrm{~mL})$, dried $\left(\mathrm{MgSO}_{4}\right)$, and concentrated in vacuo to afford the corresponding $p$-bromobenzoate ketal $(9.40 \mathrm{~g}, 97 \%)$ as a white solid.

32

$R_{\mathrm{f}}=0.49$ (silica gel, hexane/EtOAc 2:1); IR (film) $v_{\max }=1720,1590,1460,1374$, 1270, 1099, 1011, $755 \mathrm{~cm}^{-1} ;{ }^{1} \mathrm{H}$ NMR $\left(500 \mathrm{MHz}, \mathrm{CDCl}_{3}\right): \delta=7.89(\mathrm{~d}, J=8.4 \mathrm{~Hz}, 2 \mathrm{H}), 7.58(\mathrm{~d}$, $J=8.8 \mathrm{~Hz}, 2 \mathrm{H}), 7.19(\mathrm{t}, J=8.1 \mathrm{~Hz}, 1 \mathrm{H}), 7.00(\mathrm{~d}, J=7.3 \mathrm{~Hz}, 1 \mathrm{H}), 6.84(\mathrm{~d}, J=8.4 \mathrm{~Hz}, 1 \mathrm{H})$, $5.23(\mathrm{~s}, 2 \mathrm{H}), 4.94$ (s, $2 \mathrm{H}), 1.55$ (s, $6 \mathrm{H}) \mathrm{ppm} ;{ }^{13} \mathrm{C}$ NMR $\left(125 \mathrm{MHz}, \mathrm{CDCl}_{3}\right): \delta=165.5,151.5$, 132.4, 131.8 (2 C), 131.1 (2 C), 128.6, 128.4, 128.1, 121.4, 118.5, 117.8, 99.1, 64.0, 59.1, 24.6 (2 C) ppm; HRMS (MALDI): calcd for $\mathrm{C}_{18} \mathrm{H}_{17} \mathrm{O}_{4} \mathrm{Br}[\mathrm{MH}]^{+}:$399.0202, found: 399.0200 .

To a mixture of the $p$-bromo benzoate ketal $(9.40 \mathrm{~g}, 25.0 \mathrm{mmol})$, prepared above, in THF: $\mathrm{H}_{2} \mathrm{O}$ $(1.5: 1,350 \mathrm{~mL})$ was added $p-\mathrm{TSOH} \cdot \mathrm{H}_{2} \mathrm{O}(1.42 \mathrm{~g}, 7.5 \mathrm{mmol})$. The mixture was then heated for $10 \mathrm{~h}$ at $75^{\circ} \mathrm{C}$. The mixture was then diluted with EtOAc $(150 \mathrm{~mL})$ and the organic phase washed with saturated aq. $\mathrm{NaHCO}_{3}(200 \mathrm{~mL})$. The solution was dried $\left(\mathrm{MgSO}_{4}\right)$ and concentrated in vacuo to afford the corresponding hydroxy phenol $(7.14 \mathrm{~g}, 85 \%)$ as white crystals.

M.p. $=145{ }^{\circ} \mathrm{C}\left(\right.$ EtOAc); $R_{\mathrm{f}}=0.15$ (silica gel, hexane/EtOAc 2:1); IR (film) $v_{\max }=3100,1703$, 1589, 1467, 1396, 1276, 1117, 1011, $755 \mathrm{~cm}^{-1} ;{ }^{1} \mathrm{H}$ NMR $\left(500 \mathrm{MHz}, \mathrm{CDCl}_{3}\right): \delta=7.87$ (d, $J=8.8$ $\mathrm{Hz}, 2 \mathrm{H}), 7.56(\mathrm{~d}, J=8.6 \mathrm{~Hz}, 2 \mathrm{H}), 7.21(\mathrm{t}, J=7.8 \mathrm{~Hz}, 1 \mathrm{H}), 6.97$ (d, $J=7.3 \mathrm{~Hz}, 1 \mathrm{H}), 6.93$ (d, $J$ $=8.0 \mathrm{~Hz}, 1 \mathrm{H}), 5.32(\mathrm{~s}, 2 \mathrm{H}), 5.03(\mathrm{~s}, 2 \mathrm{H}) \mathrm{ppm} ;{ }^{13} \mathrm{C} \mathrm{NMR}\left(125 \mathrm{MHz}, \mathrm{CDCl}_{3}\right): \delta=165.5,156.9$, 133.3, 131.8 (2 C), 131.1 (2 C), 129.2, 128.7, 128.4, 123.7, 122.1, 117.7, 65.0, 60.0 ppm; HRMS (MALDI): calcd for $\mathrm{C}_{15} \mathrm{H}_{13} \mathrm{O}_{4} \mathrm{Br}$ [MNa] ${ }^{+}: 358.9889$, found: 358.9898 .

A solution of the above prepared hydroxy phenol $(7.14 \mathrm{~g}, 21.25 \mathrm{mmol})$ in EtOAc $(250 \mathrm{~mL})$ was treated with $\mathrm{MnO}_{2}(18.48 \mathrm{~g}, 212.5 \mathrm{mmol})$. The reaction mixture was stirred for $1.5 \mathrm{~h}$ at $25^{\circ} \mathrm{C}$ and then filtered through a pad of celite. The solvent was removed in vacuo and the crude residue was purified by flash column chromatography (silica gel, EtOAc:hexane 1:8 $\rightarrow 1: 3$ ) to afford phenolic aldehyde $32(6.15 \mathrm{~g}, 87 \%)$ as white crystals.

32: M.p. $133-135^{\circ} \mathrm{C}$ (EtOAc); $R_{\mathrm{f}}=0.43$ (silica gel, hexane/EtOAc 2:1); IR (film) $v_{\max }=1727$, 1643, 1587, 1453, 1267, 1165, 1099, 1069, 1010, 842, 803, 752, $712 \mathrm{~cm}^{-1} ;{ }^{1} \mathrm{H}$ NMR $(500 \mathrm{MHz}$, $\left.\mathrm{CDCl}_{3}\right): \delta=11.91(\mathrm{~s}, 1 \mathrm{H}), 10.38(\mathrm{~s}, 1 \mathrm{H}), 7.86(\mathrm{~d}, J=8.7 \mathrm{~Hz}, 2 \mathrm{H}), 7.56(\mathrm{~d}, J=8.3 \mathrm{~Hz}, 2 \mathrm{H})$, $7.50(\mathrm{dd}, J=7.8,7.4 \mathrm{~Hz}, 1 \mathrm{H}), 7.03(\mathrm{~d}, J=7.4 \mathrm{~Hz}, 1 \mathrm{H}), 7.02(\mathrm{~d}, J=8.2 \mathrm{~Hz}, 1 \mathrm{H}), 5.62(\mathrm{~s}, 2 \mathrm{H})$ ppm; ${ }^{13} \mathrm{C}$ NMR $\left(125 \mathrm{MHz}, \mathrm{CDCl}_{3}\right): \delta=194.8,165.1,163.4,138.3,137.2,131.8$ (2 C), $131.1(2$ 
C), 128.6, 128.2, 121.7, 119.4, 117.4, 63.4 ppm; HRMS (MALDI): calcd for $\mathrm{C}_{15} \mathrm{H}_{11} \mathrm{O}_{4} \mathrm{Br}$ $[\mathrm{MNa}]^{+}: 356.9733$, found: 356.9743 .

\section{Acyl cyanide 41 (Scheme 8a):}

To a solution of $32(3.0 \mathrm{~g}, 8.98 \mathrm{mmol})$ in anhydrous acetone $(100 \mathrm{~mL})$ was added finely powdered $\mathrm{K}_{2} \mathrm{CO}_{3}(2.48 \mathrm{~g}, 17.96 \mathrm{mmol}$ ) followed by 3-bromo-2-methyl-propene (1.82 g, 13.47 Me $\mathrm{mmol}$ ). The reaction mixture was heated to reflux for $2 \mathrm{~h}$. It is important to note that the $R_{\mathrm{f}}$ value (silica gel, hexane/EtOAc 2:1) of the reaction product is identical to that of the starting material 32. Filtration through a pad of celite followed by evaporation of the solvent in vacuo afforded the requisite alkylated benzaldehyde ( $3.17 \mathrm{~g}, 91 \%)$ as white crystals.

M.p. $86-87^{\circ} \mathrm{C}$ (acetone); $R_{\mathrm{f}}=0.43$ (silica gel, hexane/EtOAc 2:1); IR (film) $v_{\max }=1720,1684$, $1584,1473,1393,1274,1172,1121,1071,895,770,751 \mathrm{~cm}^{-1} ;{ }^{1} \mathrm{H} \mathrm{NMR}\left(500 \mathrm{MHz}, \mathrm{CDCl}_{3}\right): \delta=$ 10.72 (s, $1 \mathrm{H}), 7.96$ (d, $J=8.8 \mathrm{~Hz}, 2 \mathrm{H}), 7.86$ (d, $J=8.7 \mathrm{~Hz}, 2 \mathrm{H}), 7.50$ (t, $J=7.9, \mathrm{~Hz}, 1 \mathrm{H}), 7.12$ (d, $J=7.9 \mathrm{~Hz}, 1 \mathrm{H}), 6.96(\mathrm{~d}, J=8.3 \mathrm{~Hz}, 1 \mathrm{H}), 5.75(\mathrm{~s}, 2 \mathrm{H}), 5.11(\mathrm{~s}, 1 \mathrm{H}), 5.04(\mathrm{~s}, 1 \mathrm{H}), 4.55$ (s, 2 $\mathrm{H}), 1.85$ (s, $3 \mathrm{H}) \mathrm{ppm} ;{ }^{13} \mathrm{C}$ NMR $\left(125 \mathrm{MHz}, \mathrm{CDCl}_{3}\right): \delta=191.4,165.3,162.3,139.8,139.5$, 135.1, 131.7 (2 C), 131.2 (2 C), 128.9, 128.1, 122.0, 119.0, 113.4, 112.2, 72.4, 64.9, 19.4 ppm; HRMS (MALDI): calcd for $\mathrm{C}_{19} \mathrm{H}_{17} \mathrm{O}_{4} \mathrm{Br}$ [MNa ${ }^{+}$: 411.0202 , found: 411.0214 .

A solution of $\mathrm{Et}_{2} \mathrm{AlCN}$ ( $8.98 \mathrm{~mL}, 1 \mathrm{M}$ in toluene) was added slowly to a solution of the alkylated benzaldehyde $(3.17 \mathrm{~g}, 8.17 \mathrm{mmol})$, prepared above, in toluene $(70 \mathrm{~mL})$ at $0{ }^{\circ} \mathrm{C}$. After $30 \mathrm{~min}$ stirring at the same temperature, the reaction solution was warmed to $25{ }^{\circ} \mathrm{C}$ and stirred for a further $30 \mathrm{~min}$. Saturated aq. sodium potassium tartrate $(50 \mathrm{~mL})$ was added and the emulsion was stirred vigorously for $1 \mathrm{~h}$. The layers were separated and the organic phase was dried with $\mathrm{MgSO}_{4}$ and concentrated in vacuo. The crude residue was purified by flash column chromatography (silica gel, EtOAc:hexane 1:6 $\rightarrow 1: 4)$ to provide the desired cyanohydrin $(2.71$ $\mathrm{g}, 82 \%)$ as a viscous syrup.

$R_{\mathrm{f}}=0.28$ (silica gel, hexane/EtOAc 2:1); IR (film) $v_{\max }=3430,3086,2975,2937,1720,1589$, 1473, 1450, 1397, 1377, 1268, 1101, 1011, 916, $756 \mathrm{~cm}^{-1} ;{ }^{1} \mathrm{H}$ NMR $\left(500 \mathrm{MHz}, \mathrm{CDCl}_{3}\right): \delta=7.92$ $(\mathrm{d}, J=8.5 \mathrm{~Hz}, 2 \mathrm{H}), 7.57$ (d, $J=8.8 \mathrm{~Hz}, 2 \mathrm{H}), 7.38$ (dd, $J=8.5,7.7, \mathrm{~Hz}, 1 \mathrm{H}), 7.11$ (dd, $J=7.7$, $0.7 \mathrm{~Hz}, 1 \mathrm{H}), 7.03(\mathrm{~d}, J=8.5 \mathrm{~Hz}, 1 \mathrm{H}), 5.85(\mathrm{~d}, J=11.4 \mathrm{~Hz}, 1 \mathrm{H}), 5.43($ AB system $\Delta \delta=0.0374$ ppm, $J=12.8 \mathrm{~Hz}, 2 \mathrm{H}$ ), 5.17 (s, $1 \mathrm{H}), 5.09(\mathrm{~s}, 1 \mathrm{H}), 4.63$ (AB system $\Delta \delta=0.0476 \mathrm{ppm}, J=12.1$ 
$\mathrm{Hz}, 2 \mathrm{H}), 4.45(\mathrm{~d}, J=11.4 \mathrm{~Hz}, 1 \mathrm{H}) 1.91(\mathrm{~s}, 3 \mathrm{H}) \mathrm{ppm} ;{ }^{13} \mathrm{C} \mathrm{NMR}\left(125 \mathrm{MHz}, \mathrm{CDCl}_{3}\right): \delta=165.5$, 157.1, 139.4, 134.4, 131.9 (2 C), 131.3 (2 C), 131.0, 128.7, 128.2, 123.7, 123.6, 119.1, 114.3, 113.5, 72.9, 64.7, 57.9, 19.6 ppm; HRMS (MALDI): calcd for $\mathrm{C}_{20} \mathrm{H}_{18} \mathrm{O}_{4} \mathrm{NBr}[\mathrm{MNa}]^{+}: 438.0311$, found: 438.0305 .

A solution of the cyanohydrin $(2.17 \mathrm{~g}, 6.70 \mathrm{mmol})$, prepared above, in anhydrous $\mathrm{CH}_{2} \mathrm{Cl}_{2}(90$ $\mathrm{mL})$ was heated to reflux for $12 \mathrm{~h}$ in the presence of PCC (5.77 $\mathrm{g}, 26.80 \mathrm{mmol})$. A sufficient amount of celite was then added to absorb the reaction mixture accompanied by vigorous stirring. The resulting paste was transferred directly on to the top of a silica gel column and subsequent flash column chromatography (EtOAc:hexane 1:6 $\rightarrow$ 1:3) furnished acyl cyanide 41 $(2.02 \mathrm{~g}, 73 \%)$ as white crystals.

41: M.p. $93-95{ }^{\circ} \mathrm{C}\left(\right.$ EtOAc); $R_{\mathrm{f}}=0.41$ (silica gel, hexane/EtOAc 2:1); IR (film) $v_{\max }=3079$, 2949, 1720, 1650, 1592, 1474, 1453, 1268, 1102, 1069, 1011, 980, 918, $754 \mathrm{~cm}^{-1} ;{ }^{1} \mathrm{H}$ NMR (500 $\left.\mathrm{MHz}_{\mathrm{CDCl}}\right): \delta=7.92(\mathrm{~d}, J=8.7 \mathrm{~Hz}, 2 \mathrm{H}), 7.59(\mathrm{~d}, J=8.7 \mathrm{~Hz}, 2 \mathrm{H}), 7.56(\mathrm{dd}, J=8.7,7.8 \mathrm{~Hz}$, $1 \mathrm{H}), 7.16(\mathrm{~d}, J=7.8 \mathrm{~Hz}, 1 \mathrm{H}), 7.04(\mathrm{~d}, J=8.7 \mathrm{~Hz}, 1 \mathrm{H}), 5.46$ (s, $2 \mathrm{H}), 5.15$ (s, $1 \mathrm{H}), 5.08$ (s, 1 $\mathrm{H}), 4.66(\mathrm{~s}, 2 \mathrm{H}), 1.90(\mathrm{~s}, 3 \mathrm{H}) \mathrm{ppm} ;{ }^{13} \mathrm{C} \mathrm{NMR}\left(125 \mathrm{MHz}, \mathrm{CDCl}_{3}\right): \delta=167.6,165.2,160.2$, 139.4, 139.3, 135.9, 131.8 (2 C), 131.2 (2 C), 128.5, 128.4, 122.4, 121.1, 114.6, 114.4, 113.1, 73.5, 63.9, 19.5 ppm; HRMS (MALDI): calcd for $\mathrm{C}_{20} \mathrm{H}_{16} \mathrm{O}_{4} \mathrm{NBr}[\mathrm{MNa}]^{+}$: 436.0155, found: 436.0160 .

The same protocol was applied for the following transformations: $\mathbf{2 7} \rightarrow \mathbf{2 8}$ (Scheme 5), $\mathbf{3 5} \rightarrow \mathbf{3 6}$ (Scheme 6), and $\mathbf{3 3} \rightarrow \mathbf{3 8}$ (Scheme 7).

General procedure for the coupling of acyl cyanides 28, 38, and 41 with 1,3cyclohexadiones 5, 7a, 7b, 8a, and $8 b$ (Scheme 8a):

To a solution of acyl cyanide $41(1.0 \mathrm{~g}, 2.42 \mathrm{mmol})$ in THF (25 mL) was added the appropriate 1,3-cyclohexadione 5 or $7 \mathbf{a}$ or $\mathbf{7 b}(2.90 \mathrm{mmol})$ followed by $\mathrm{Et}_{3} \mathrm{~N}(0.67 \mathrm{~mL}, 4.84 \mathrm{mmol})$. The resulting solution was stirred for 6-12 h (until complete consumption of the starting acyl cyanide 41 by TLC) at $25^{\circ} \mathrm{C}$. The solution was diluted with $\mathrm{Et}_{2} \mathrm{O}(50 \mathrm{~mL})$, washed with $1 \mathrm{M} \mathrm{HCl}(50$ $\mathrm{mL})$ and brine $(50 \mathrm{~mL})$ and dried $\left(\mathrm{MgSO}_{4}\right)$. The solvent was removed in vacuo and the residual crude syrup was purified by flash column chromatography (silica gel, EtOAc:hexane 1:6 $\rightarrow$ 1:2) furnishing pure tricarbonyls $\mathbf{4 2 a}, \mathbf{b}$, and $\mathbf{c}(91-98 \%)$ as syrups. 
42a: $R_{\mathrm{f}}=0.40$ (silica gel, hexane/EtOAc 2:1); IR (film) $v_{\max }=2956,1722,1676,1589,1556$, 1469, 1453, 1398, 1267, 1101, 1069, 1011, 882, $756 \mathrm{~cm}^{-1} ;{ }^{1} \mathrm{H}$ NMR $\left(600 \mathrm{MHz}, \mathrm{CDCl}_{3}\right): \delta=$ $17.34(\mathrm{~s}, 1 \mathrm{H}), 7.81(\mathrm{~d}, J=8.8 \mathrm{~Hz}, 2 \mathrm{H}), 7.53(\mathrm{~d}, J=7.7 \mathrm{~Hz}, 2 \mathrm{H}), 7.34(\mathrm{t}, J=7.7 \mathrm{~Hz}, 1 \mathrm{H}), 7.08$ $(\mathrm{d}, J=7.7 \mathrm{~Hz}, 1 \mathrm{H}), 6.87$ (d, J= 7.7 Hz, 1 H), 5.36 (brs, $1 \mathrm{H}), 5.16$ (brs, $1 \mathrm{H}), 4.94$ (s, $1 \mathrm{H}), 4.89$ (s, 1 H), 4.37 (brs, 1 H), 4.34 (brs, 1 H), 2.64 (brs, 1 H), 2.45 - 2.00 (brm, 4 H), 1.70 (s, 3 H), 1.01 (brd, $J=5.5 \mathrm{~Hz}, 3 \mathrm{H}$ ) ppm; ${ }^{13} \mathrm{C} \mathrm{NMR}\left(150 \mathrm{MHz}, \mathrm{CDCl}_{3}\right): \delta=198.1$ (br), 195.8, 193.2 (br), 165.2, 161.6 (br), 154.9 (br), 140.4, 132.8 (br), 131.6 (2 C), 131.2 (2 C), 130.1, 128.9, 128.8, 128.0, 121.5, 114.5, 112.1, 72.0, 64.6, 46.1 (br), 40.3, 26.4, 20.8, 19.2 ppm; HRMS (MALDI): calcd for $\mathrm{C}_{26} \mathrm{H}_{25} \mathrm{O}_{6} \mathrm{Br}\left[\mathrm{MNa}^{+}\right]$: 535.0727, found: 535.0719.

The same protocol was applied to the coupling of acyl cyanide $\mathbf{2 8}$ with cyclohexadione $\mathbf{5}$ or $\mathbf{7 b}$ (Scheme 5) and for the coupling of acyl cyanide 38 with $\mathbf{7 b}$ (Scheme 7). In the case of bisalkylated 1,3-cyclohexadiones $8 \mathbf{a}$ or $\mathbf{8 b}$, addition of 1.0 equiv. of 4-DMAP and longer reaction times (72-96 h) proved to be necessary for the formation of ticarbonyles $\mathbf{4 4 a}$, and $\mathbf{b}$ (83-86\%, Scheme 8B). 4-DMAP was also used for the coupling of 41 with $65 \mathbf{a}$, or $\mathbf{b}$ (Scheme 13). Compounds $\mathbf{4 2 b}, \mathbf{4 2 c}, \mathbf{4 4 a}$, and 44b appear in the NMR spectra as complicated mixtures of at least four tautomeric atropisomers making their full spectral analysis difficult.

\section{Preparation of $\Delta^{16,17}(Z)$-macrocyclic compound 43 (Scheme 8):}

To a degassed solution of $\mathbf{4 2 b}$ or $\mathbf{4 2 c}(0.082 \mathrm{mmol}$, bubbling for $15 \mathrm{~min}$ with Ar) in anhydrous $\mathrm{CH}_{2} \mathrm{Cl}_{2}(5 \mathrm{~mL})$ was added the second generation Grubbs catalyst $\mathbf{A}$ (14.0 $\mathrm{mg}, 0.0164 \mathrm{mmol}$ or

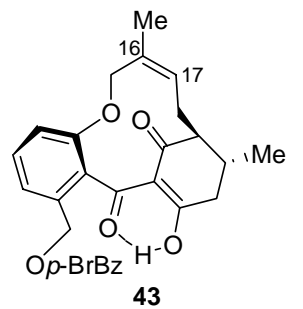
$21.0 \mathrm{mg}, 0.0247 \mathrm{mmol}$, respectively) in one portion. The resulting pink solution was heated to reflux, with light excluded from the reaction vessel, for 5 or $18 \mathrm{~h}$, respectively. The solvent was removed in vacuo and the residue was purified by flash column chromatography (silica gel, EtOAc:hexane 1:8 $\rightarrow$ $1: 2)$ to afford $\Delta^{16,17}(Z)$-macrocycle $43(25.8 \mathrm{mg}, 60 \%$ or $12.9 \mathrm{mg}, 30 \%$, respectively) as a white crystalline solid. The structure of $\mathbf{4 3}$ was unambiguously assigned by $\mathrm{x}-$ ray analysis (Ref. 28).

43: M.p. $184-186{ }^{\circ} \mathrm{C}\left(\right.$ EtOAc); $R_{\mathrm{f}}=0.41$ (silica gel, hexane/EtOAc 2:1); IR (film) $v_{\max }=2961$, $1721,1668,1589,1456,1412,1266,1115,1103,1068,1011,757,735 \mathrm{~cm}^{-1} ;{ }^{1} \mathrm{H}$ NMR $(500$ $\mathrm{MHz}, \mathrm{CDCl}_{3}$ ): $\delta=16.57$ (brs, $\left.1 \mathrm{H}\right), 7.83$ (d, $\left.J=8.3 \mathrm{~Hz}, 2 \mathrm{H}\right), 7.49$ (d, $\left.J=9.1 \mathrm{~Hz}, 2 \mathrm{H}\right), 7.39$ (dd, $J=8.3,7.3 \mathrm{~Hz}, 1 \mathrm{H}), 7.15(\mathrm{~d}, J=7.3 \mathrm{~Hz}, 1 \mathrm{H}), 6.92(\mathrm{~d}, J=8.3 \mathrm{~Hz}, 1 \mathrm{H}), 5.61$ (brs, $1 \mathrm{H}), 5.60$ (d, 
$J=12.9 \mathrm{~Hz}, 1 \mathrm{H}), 5.27$ (d, $J=12.9 \mathrm{~Hz}, 1 \mathrm{H}), 4.42$ (brs, $1 \mathrm{H}), 4.15$ (brs, $1 \mathrm{H}), 2.90$ - 2.30 (brm, 4 $\mathrm{H}), 2.25-2.10$ (brm, $2 \mathrm{H}), 1.90$ (s, $3 \mathrm{H}), 1.09$ (brs, 3H) ppm; ${ }^{13} \mathrm{C} \mathrm{NMR}\left(125 \mathrm{MHz}, \mathrm{CDCl}_{3}\right): \delta=$ 197.2 (br), 195.0 (br), 191.9 (br), 171.1, 165.2, 135.4 (br), 131.4 (2 C), 131.2 (2 C), 128.9, 127.9, 127.6 (br), 122.4 (br), 115.5, 76.4, 64.8, 60.4, 54.4 (br), 24.4 (br), 21.0, 19.5 (br), 14.2 ppm. Most of the peaks in the NMR spectra are broadened because of the fast tautomeric atropisomerism, some were, therefore, difficult to assign regardless of the sample concentration. The same problem was faced when using different NMR solvents like $\mathrm{C}_{6} \mathrm{D}_{6}$ and THF- $d_{8}$, while the compound is not very soluble in $\mathrm{CD}_{3} \mathrm{CN}$ and acetone- $d_{6}$. As a result, we report 23 out of the expected 27 carbon signals; HRMS (MALDI): calcd for $\mathrm{C}_{27} \mathrm{H}_{25} \mathrm{O}_{6} \mathrm{Br}[\mathrm{MNa}]^{+}:$547.0727, found: 547.0720 .

\section{Esterification of tricarbonyl compound 42a to vinylogous esters 46 and 47 (Scheme 9):}

To a solution of $\mathbf{4 2 a}(0.57 \mathrm{~g}, 1.11 \mathrm{mmol})$ in $\mathrm{Et}_{2} \mathrm{O}(20 \mathrm{~mL})$, at $0{ }^{\circ} \mathrm{C}$, was added slowly a preprepared solution of anhydrous $\mathrm{CH}_{2} \mathrm{~N}_{2}$ in $\mathrm{Et}_{2} \mathrm{O}$ (prepared using the Aldrich protocol) until the color of the solution remained constantly pale yellow. The formation of a white precipitate was observed. The solution was stirred for $30 \mathrm{~min}$ at the same temperature. The excess $\mathrm{CH}_{2} \mathrm{~N}_{2}$ was removed by bubbling argon through the reaction mixture for $10 \mathrm{~min}$ at $25{ }^{\circ} \mathrm{C}$. The white crystalline solid was isolated by filtration and proved to be vinylogous ester 47 . The mother liquor was concentrated in vacuo and the residue was purified by flash column chromatography (silica gel, $\mathrm{CH}_{2} \mathrm{Cl}_{2} \rightarrow \mathrm{CH}_{2} \mathrm{Cl}_{2}$ :EtOAc 2:1) to afford the regioisomeric vinylogous ester 46 (0.28 $\mathrm{g}, 48 \%)$ as a syrup, plus some further quantities of crystalline 47 (0.28 g, 48\%, total).

46 (mixture of two atropisomers in 1:1 ratio in $\mathrm{CDCl}_{3}$ ): $R_{\mathrm{f}}=0.23$ (silica gel, hexane/EtOAc 2:1); IR (film) $v_{\max }=2953,1722,1650,1589,1519,1454,1267,1115,1011,757 \mathrm{~cm}^{-1} ;{ }^{1} \mathrm{H}$ NMR $(600$

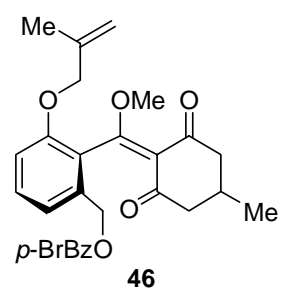
$\left.\mathrm{MHz}_{\mathrm{CDCl}}\right): \delta=7.91(\mathrm{~d}, J=8.8 \mathrm{~Hz}, 2 \mathrm{H}), 7.89(\mathrm{~d}, J=7.7 \mathrm{~Hz}, 2 \mathrm{H}), 7.58(\mathrm{~d}$, $J=8.8 \mathrm{~Hz}, 2 \mathrm{H}), 7.57(\mathrm{~d}, J=8.8 \mathrm{~Hz}, 2 \mathrm{H}), 7.40(\mathrm{dd}, J=8.7,7.7 \mathrm{~Hz}, 1 \mathrm{H})$, 7.39 (t, $J=7.7 \mathrm{~Hz}, 1 \mathrm{H}), 7.13(\mathrm{~d}, J=7.7 \mathrm{~Hz}, 1 \mathrm{H}), 7.12(\mathrm{~d}, J=7.7 \mathrm{~Hz}, 1 \mathrm{H})$, $6.92(\mathrm{t}, J=8.8 \mathrm{~Hz}, 2 \mathrm{H}), 5.30(\mathrm{~d}, J=13.1 \mathrm{~Hz}, 1 \mathrm{H}), 5.24(\mathrm{~d}, J=13.1 \mathrm{~Hz}, 1 \mathrm{H})$, $5.20(\mathrm{~d}, J=12.1 \mathrm{~Hz}, 1 \mathrm{H}), 5.18(\mathrm{~d}, J=12.1 \mathrm{~Hz}, 1 \mathrm{H}), 5.00(\mathrm{~s}, 1 \mathrm{H}), 4.99$ (s, 1 $\mathrm{H}), 4.93(\mathrm{~s}, 2 \mathrm{H}), 4.43(\mathrm{AB}$ system, $\Delta \delta=0.0274 \mathrm{ppm}, J=12.1 \mathrm{~Hz}, 2 \mathrm{H}), 4.41(\mathrm{~s}, 2 \mathrm{H}), 3.64(\mathrm{~s}, 3$ H), $3.63(\mathrm{~s}, 3 \mathrm{H}), 2.67(\mathrm{~m}, 2 \mathrm{H}), 2.43(\mathrm{~m}, 2 \mathrm{H}), 2.30-2.05(\mathrm{~m}, 6 \mathrm{H}), 1.73(\mathrm{~s}, 6 \mathrm{H}), 1.01(\mathrm{~d}, J=$ $6.6 \mathrm{~Hz}, 3 \mathrm{H}) 1.00(\mathrm{~d}, J=6.6 \mathrm{~Hz}, 3 \mathrm{H}) \mathrm{ppm} ;{ }^{13} \mathrm{C} \mathrm{NMR}\left(150 \mathrm{MHz}, \mathrm{CDCl}_{3}\right): \delta=197.0,196.9,196.4$, 
196.2, 174.9, 174.8, 165.4 (2 C), 154.9, 154.4, 140.0 (2 C), 135.6, 134.7, 131.8 (4 C), 131.2 (2 C), 131.2 (2 C), 131.0 (2 C), 128.7, 128.6, 128.4, 128.3, 122.1, 121.8, 121.3, 121.1, 118.8 (2 C), 112.5, 112.5, 112.1, 112.0, 72.1, 72.0, 64.7, 64.7, 57.5, 57.5, 48.7, 48.6, 48.0, 47.9, 25.4, 25.3, 20.9, 20.8, 19.2 (2 C) ppm; HRMS (MALDI): calcd for $\mathrm{C}_{27} \mathrm{H}_{27} \mathrm{O}_{6} \mathrm{Br}[\mathrm{MNa}]^{+}:$549.0883, found: 549.0876.

47: M.p. $165-167{ }^{\circ} \mathrm{C}\left(\mathrm{Et}_{2} \mathrm{O}\right) ; R_{\mathrm{f}}=0.11$ (silica gel, hexane/EtOAc 2:1); IR (film) $v_{\max }=1718$, 1675, 1646, 1589, 1458, 1376, 1333, 1287, 1103, 1070, 1010, $757 \mathrm{~cm}^{-1}$; ${ }^{1} \mathrm{H}$ NMR (600 MHz,

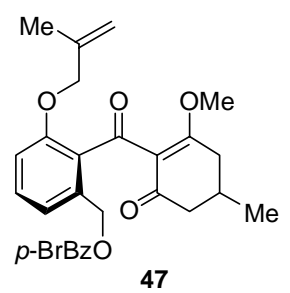
$\left.\mathrm{CDCl}_{3}\right): \delta=7.94(\mathrm{~d}, J=8.8 \mathrm{~Hz}, 2 \mathrm{H}), 7.55(\mathrm{~d}, J=7.7 \mathrm{~Hz}, 2 \mathrm{H}), 7.29$ (dd, $J=$ 8.8, $7.7 \mathrm{~Hz}, 1 \mathrm{H}), 7.09$ (d, J=7.7 Hz, $1 \mathrm{H}), 6.81$ (d, $J=8.8 \mathrm{~Hz}, 1 \mathrm{H}), 5.46$ (s, $2 \mathrm{H}), 4.98(\mathrm{~s}, 1 \mathrm{H}), 4.95(\mathrm{~s}, 1 \mathrm{H}), 4.36$ (AB system, $\Delta \delta=0.0274 \mathrm{ppm}, J=12.1$ Hz, 2 H), 3.72 (s, 3 H), 2.66 (m, 1 H), 2.34 (dd, $J=14.3,3.2$ Hz, $1 \mathrm{H}), 2.15$ $(\mathrm{m}, 2 \mathrm{H}), 2.00(\mathrm{dd}, J=16.0,12.6 \mathrm{~Hz}, 1 \mathrm{H}), 1.76(\mathrm{~s}, 3 \mathrm{H}), 1.07$ (d, $J=5.5 \mathrm{~Hz}$, 3H) $\mathrm{ppm} ;{ }^{13} \mathrm{C}$ NMR $\left(150 \mathrm{MHz}, \mathrm{CDCl}_{3}\right): \delta=195.3,194.7,176.5,165.5,156.2,140.8,136.2$, 131.5 (2 C), 131.3 (2 C), 130.6 (2 C), 129.3, 127.8, 122.2, 120.9, 112.8, 111.9, 72.4, 64.8, 56.9, 45.0, 34.5, 27.4, 21.0, 19.4 ppm; HRMS (MALDI): calcd for $\mathrm{C}_{27} \mathrm{H}_{27} \mathrm{O}_{6} \mathrm{Br}[\mathrm{MNa}]^{+}$: 549.0883, found: 549.0891 .

\section{Preparation of $\Delta^{16,17}(E)$-macrocycle 49 (Scheme 9).}

A solution of vinylogous ester $46(0.15 \mathrm{~g}, 0.285 \mathrm{mmol})$ in anhydrous THF $(2.0 \mathrm{~mL})$, containing anhydrous HMPA $(0.2 \mathrm{~mL})$, was treated with LiHMDS $\left(0.3 \mathrm{~mL}, 1 \mathrm{M}\right.$ in THF) at $-78^{\circ} \mathrm{C}$ for $1 \mathrm{~h}$.

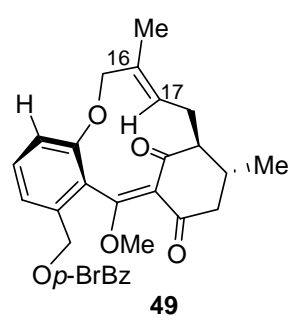

A solution of prenyl bromide $(66 \mu \mathrm{L}, 0.57 \mathrm{mmol})$ in anhydrous THF $(0.5 \mathrm{~mL})$ was then added drop-wise to the solution, at the same temperature. The resulting solution was allowed to warm slowly to $0{ }^{\circ} \mathrm{C}$ over a period of $5 \mathrm{~h}$. The solution was diluted with $\mathrm{Et}_{2} \mathrm{O}(10 \mathrm{~mL})$ and quenched with saturated aq. $\mathrm{NH}_{4} \mathrm{Cl}(5 \mathrm{~mL})$. The layers were separated and the organic phase was dried $\left(\mathrm{MgSO}_{4}\right)$ and concentrated in vacuo. The residue was purified by flash column chromatography (silica gel, EtOAc:hexane 1:4) to afford $48(0.107 \mathrm{~g}, 63 \%)$ as a syrup. Based on the ${ }^{1} \mathrm{H}-\mathrm{NMR}$ spectrum, compound $\mathbf{4 8}$ was found to be a complicated mixture of four stereo- and atropisomers, as expected.

A degassed solution of $48(0.10 \mathrm{~g}, 0.17 \mathrm{mmol}$, bubbling for $15 \mathrm{~min}$ with Ar) in anhydrous $\mathrm{CH}_{2} \mathrm{Cl}_{2}(10 \mathrm{~mL})$ was treated with the second generation Grubbs catalyst A $(29.0 \mathrm{mg}, 0.034$ 
mmol). The resulting pink solution was heated to reflux, with light excluded from the reaction vessel, for $20 \mathrm{~h}$. The solvent was removed in vасио and the residue was purified by flash column chromatography (silica gel, EtOAc:hexane $1: 8 \rightarrow 1: 2)$ to afford $\Delta^{16,17}(E)$-macrocycle $49(27.4$ $\mathrm{mg}, 30 \%)$ as a syrup along with recovered starting material 48 (35 mg, 35\%). The (E)-geometry of the newly formed $\Delta^{16,17}$ macrocyclic double bond was unambiguously proved by extensive NOE experiments (see Scheme 9).

49: $R_{\mathrm{f}}=0.30$ (silica gel, hexane/EtOAc 2:1); IR (film) $v_{\max }=2955,1721,1651,1589,1531$, 1455, 1267, 1233, 1117, 1101, 1011, 849, $757 \mathrm{~cm}^{-1} ;{ }^{1} \mathrm{H}$ NMR $\left(500 \mathrm{MHz}, \mathrm{CDCl}_{3}\right): \delta=7.92(\mathrm{~d}, J$ $=8.3 \mathrm{~Hz}, 2 \mathrm{H}), 7.59(\mathrm{~d}, J=8.3 \mathrm{~Hz}, 2 \mathrm{H}), 7.40(\mathrm{dd}, J=8.3,7.3 \mathrm{~Hz}, 1 \mathrm{H}), 7.23(\mathrm{~d}, J=7.3 \mathrm{~Hz}, 1$ H), 7.19 (d, $J=8.3 \mathrm{~Hz}, 1 \mathrm{H}), 5.69$ (brdd, $J=11.0,2.7 \mathrm{~Hz}, 1 \mathrm{H}), 5.48$ (d, $J=12.9 \mathrm{~Hz}, 1 \mathrm{H}), 5.29$ $(\mathrm{d}, J=12.9 \mathrm{~Hz}, 1 \mathrm{H}), 4.51$ (AB system, $\Delta \delta=0.0384 \mathrm{ppm}, J=11.9 \mathrm{~Hz}, 2 \mathrm{H}), 3.52(\mathrm{~s}, 3 \mathrm{H}), 2.65$ - 2.57 (m, $2 \mathrm{H}), 2.26-2.17$ (m, $4 \mathrm{H}), 1.37$ (s, $3 \mathrm{H}), 1.10$ (d, J=5.5 Hz, 3H) ppm; ${ }^{13} \mathrm{C}$ NMR $(125$ $\mathrm{MHz}, \mathrm{CDCl}_{3}$ ): $\delta=201.7,195.1,173.7,165.9,155.0,139.0,135.8,132.2$ (2 C), 131.7 (2 C), $131.4,130.0,129.2$, 128.7, 125.0, 123.1, 120.5, 120.3, 65.8, 57.8, 55.9, 47.8, 32.0, 28.7, 23.1, 19.5, 14.5 ppm. HRMS (MALDI): calcd for $\mathrm{C}_{28} \mathrm{H}_{27} \mathrm{O}_{6} \mathrm{Br}\left[\mathrm{MNa}^{+}\right]: 561.0883$, found: 561.0886 .

\section{Stereospecific olefin metathesis of regioisomeric vinylogous esters 50, 51 and 52 (Scheme 10):}

To a solution of tricarbonyl compound $44 \mathrm{~b}(0.80 \mathrm{~g}, 1.23 \mathrm{mmol})$ in $\mathrm{Et}_{2} \mathrm{O}(20 \mathrm{~mL})$, at $0{ }^{\circ} \mathrm{C}$, was added slowly a pre-prepared solution of anhydrous $\mathrm{CH}_{2} \mathrm{~N}_{2}$ in $\mathrm{Et}_{2} \mathrm{O}$ (prepared using the Aldrich protocol) until the color of the solution remained constantly yellow. The solution was stirred for a further $1 \mathrm{~h}$ at the same temperature. The excess $\mathrm{CH}_{2} \mathrm{~N}_{2}$ was removed by bubbling argon through the reaction mixture for $15 \mathrm{~min}$ at $25^{\circ} \mathrm{C}$. Formation of three more polar spots, compared to the starting material $\mathbf{4 4 b}$, was observed by TLC. Separation of the products corresponding to these spots by flash column chromatography (silica gel, EtOAc:hexane 1:6 $\rightarrow$ 1:4) afforded 50 $(0.26 \mathrm{~g}, 32 \%), \mathbf{5 1}(0.39 \mathrm{~g}, 48 \%)$ and $\mathbf{5 2}(0.13 \mathrm{~g}, 16 \%)$ as syrups. Regioisomer $\mathbf{5 1}$ was a mixture of $\Delta^{8,9}$ double bond geometrical isomers (ca. 1.3:1), each of which again consisted of a pair of atropisomers (ca. 1:1). As a result the NMR spectral data for $\mathbf{5 1}$ can not easily be reported.

50: $R_{\mathrm{f}}=0.47$ (silica gel, hexane/EtOAc 2:1); IR (film) $v_{\max }=2966,2914,1721,1672,1650$, 1590, 1455, 1377, 1268, 1115, 1069, 1011, 904, 848, $757 \mathrm{~cm}^{-1} ;{ }^{1} \mathrm{H}$ NMR $\left(600 \mathrm{MHz}, \mathrm{CDCl}_{3}\right): \delta=$ $7.97(\mathrm{~d}, J=8.3 \mathrm{~Hz}, 2 \mathrm{H}), 7.54(\mathrm{~d}, J=9.1 \mathrm{~Hz}, 2 \mathrm{H}), 7.29$ (t, $J=8.2, \mathrm{~Hz}, 1 \mathrm{H}), 7.12(\mathrm{~d}, J=7.4 \mathrm{~Hz}$, 


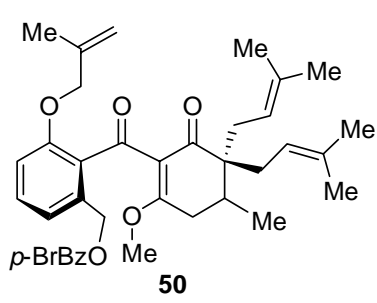

$1 \mathrm{H}), 6.79(\mathrm{~d}, J=8.2 \mathrm{~Hz}, 1 \mathrm{H}), 5.50(\mathrm{AB}$ system, $\Delta \delta=0.0299 \mathrm{ppm}, J=$ $13.1 \mathrm{~Hz}, 2 \mathrm{H}), 4.96$ (s, $1 \mathrm{H}), 4.93$ (s, $1 \mathrm{H}), 4.80$ (brt, $J=6.8 \mathrm{~Hz}, 1 \mathrm{H}$ ), 4.69 (brt, $J=6.8 \mathrm{~Hz}, 1 \mathrm{H}), 4.29$ (s, $2 \mathrm{H}), 3.76$ (s, $3 \mathrm{H}), 2.67$ (dd, $J=$ $19.2,5.5 \mathrm{~Hz}, 1 \mathrm{H}), 2.38(\mathrm{~m}, 2 \mathrm{H}), 2.23(\mathrm{~m}, 1 \mathrm{H}), 2.15(\mathrm{dd}, J=15.6,6.4$ Hz, 1 H), $2.05-1.95$ (m, 2 H), 1.75 (s, $3 \mathrm{H}), 1.60$ (s, $3 \mathrm{H}), 1.58$ (s, $3 \mathrm{H})$, $1.53(\mathrm{~s}, 3 \mathrm{H}), 1.52(\mathrm{~s}, 3 \mathrm{H}), 0.96(\mathrm{~d}, J=7.3 \mathrm{~Hz}, 3 \mathrm{H}) \mathrm{ppm} ;{ }^{13} \mathrm{C} \mathrm{NMR}\left(125 \mathrm{MHz}, \mathrm{CDCl}_{3}\right): \delta=$ 199.6, 195.7, 173.6, 165.6, 155.9, 140.9, 136.2, 133.4, 132.6, 131.5 (2 C), 131.4 (2 C), 130.9, 130.3, 129.4, 127.8, 121.3, 121.1, 120.3, 119.0, 112.5, 111.4, 71.9, 64.9, 56.2, 50.5, 31.9, 31.5, 31.3, 28.7, 26.1, 25.9, 19.4, 17.9, 17.8, 15.7 ppm; HRMS (MALDI): calcd for $\mathrm{C}_{37} \mathrm{H}_{43} \mathrm{O}_{6} \mathrm{Br}$ $[\mathrm{MH}]^{+}:$663.2316, found: 663.2330 .

52: $R_{\mathrm{f}}=0.62$ (silica gel, hexane/EtOAc 2:1); IR (film) $v_{\max }=2968,2913,1721,1659,1643$, 1589, 1453, 1378, 1322, 1268, 1115, 1011, 910, 846, 757, $733 \mathrm{~cm}^{-1} ;{ }^{1} \mathrm{H}$ NMR $(500 \mathrm{MHz}$,

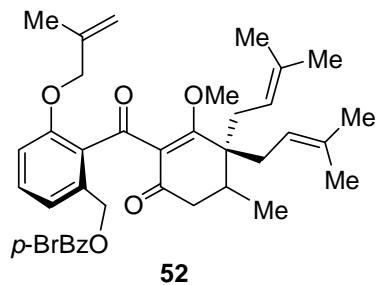

52

$\left.\mathrm{CDCl}_{3}\right): \delta=7.91(\mathrm{~d}, J=8.5 \mathrm{~Hz}, 2 \mathrm{H}), 7.51(\mathrm{~d}, J=8.5 \mathrm{~Hz}, 2 \mathrm{H}), 7.30(\mathrm{dd}$, $J=8.4,7.7 \mathrm{~Hz}, 1 \mathrm{H}), 7.13(\mathrm{~d}, J=7.7 \mathrm{~Hz}, 1 \mathrm{H}), 6.79(\mathrm{~d}, J=8.4 \mathrm{~Hz}, 1 \mathrm{H})$, 5.52 (AB system, $\Delta \delta=0.0895 \mathrm{ppm}, J=13.6 \mathrm{~Hz}, 2 \mathrm{H}$ ), 5.14 (brt, $J=6.5$ $\mathrm{Hz}, 1 \mathrm{H}$ ), 4.94 (brt, $J=6.5 \mathrm{~Hz}, 1 \mathrm{H}$ ), 4.93 (brs, $1 \mathrm{H}$ ), 4.92 (brs, $1 \mathrm{H}$ ), 4.45 (brs, $2 \mathrm{H}), 3.86$ (s, $3 \mathrm{H}), 2.75-2.50$ (m, $2 \mathrm{H}), 2.40-2.20$ (m, $3 \mathrm{H}), 2.15$ - 2.00 (m, 2 H), 1.72 (s, 3 H), 1.68 (s, 3 H), 1.65 (s, 3 H), 1.63 (s, 3 H), 1.60 (s, 3 H), 0.93 (d, $J=$ $6.6 \mathrm{~Hz}, 3 \mathrm{H}) \mathrm{ppm} ;{ }^{13} \mathrm{C} \mathrm{NMR}\left(125 \mathrm{MHz}, \mathrm{CDCl}_{3}\right): \delta=207.6,196.7,194.9,165.4,156.2,140.0$, 136.7, 134.1, 132.9, 131.5 (2 C), 131.3 (2 C), 130.9, 129.9, 129.3, 127.7, 122.8, 121.3, 120.1, 119.8, 112.5, 112.0, 72.0, 67.7, 65.1, 63.1, 55.0, 49.3, 42.3, 33.0, 26.0, 25.9, 19.1, 17.9, 17.8, 15.2 ppm; HRMS (MALDI): calcd for $\mathrm{C}_{37} \mathrm{H}_{43} \mathrm{O}_{6} \mathrm{Br}\left[\mathrm{MNa}^{+}\right]$: 685.2135, found: 685.2127.

The same protocol was used for the protection of tricarbonyl compounds 66a and $\mathbf{b}$ (Scheme 13).

To a degassed solution of regioisomeric vinylogous esters $\mathbf{5 0}$ or $\mathbf{5 1}$ or $\mathbf{5 2}(0.10 \mathrm{~g}, 0.15 \mathrm{mmol}$, bubbling for 15 min with $\mathrm{Ar}$ ) in anhydrous $\mathrm{CH}_{2} \mathrm{Cl}_{2}(10 \mathrm{~mL})$ was treated with the second generation Grubbs catalyst A (12.8 mg, $0.015 \mathrm{mmol})$, added in a single portion. The resulting pink solution was heated to reflux, with light excluded from the reaction vessel, for $3 \mathrm{~h}$ or $3 \mathrm{~h}$ or $4.5 \mathrm{~h}$, respectively. The solvent was removed in vacuo and the residue was purified by flash column chromatography (silica gel, EtOAc:hexane 1:8 $\rightarrow 1: 3$ ) to afford 11-membered $\Delta^{16,17}(Z)$ - 
macrocycle $53(73 \mathrm{mg}, 80 \%)$ as a white crystalline solid, or $\Delta^{16,17}(E)$-macrocycle 54 (78 mg, $86 \%)$ as syrup, or $\Delta^{16,17}(Z)$-macrocycle $\mathbf{5 5}(61 \mathrm{mg}, 67 \%)$ as a syrup. The structure of $\mathbf{5 3}$ was unambiguously assigned by $\mathrm{x}$-ray analysis (Ref. 32) while the $\Delta^{16,17}$ double bond geometry of $\mathbf{5 5}$ was assigned by extensive NOE experiments (see Scheme 10).

53: M.p. $192-194{ }^{\circ} \mathrm{C}$ (hexane/EtOAc 3:1); $R_{\mathrm{f}}=0.32$ (silica gel, hexane/EtOAc 2:1); IR (film) $v_{\max }=2917,1721,1682,1651,1621,1590,1469,1378,1321,1268,1236,1173,1115,1068$,

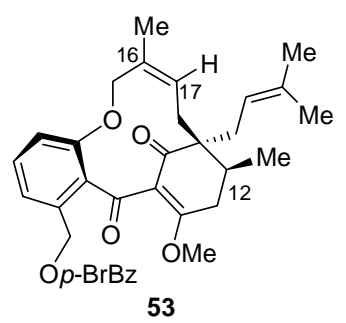

$1011,990,910,848,757,731 \mathrm{~cm}^{-1} ;{ }^{1} \mathrm{H}$ NMR $\left(500 \mathrm{MHz}, \mathrm{CD}_{3} \mathrm{CN}\right): \delta=7.94$ (d, $J=9.1 \mathrm{~Hz}, 2 \mathrm{H}), 7.66(\mathrm{~d}, J=9.1 \mathrm{~Hz}, 2 \mathrm{H}), 7.37(\mathrm{t}, J=8.2 \mathrm{~Hz}, 1 \mathrm{H}), 7.12$ (d, $J=8.2 \mathrm{~Hz}, 1 \mathrm{H}), 7.04(\mathrm{~d}, J=8.2 \mathrm{~Hz}, 1 \mathrm{H}), 5.59(\mathrm{dd}, J=11.9,4.6 \mathrm{~Hz}, 1$ H), $5.43(\mathrm{~d}, J=13.7 \mathrm{~Hz}, 1 \mathrm{H}), 5.33(\mathrm{~d}, J=13.7 \mathrm{~Hz}, 1 \mathrm{H}), 4.86(\mathrm{brm}, 1 \mathrm{H})$, $4.52(\mathrm{~d}, J=11.0 \mathrm{~Hz}, 1 \mathrm{H}), 4.34(\mathrm{~d}, J=11.0 \mathrm{~Hz}, 1 \mathrm{H}), 3.53(\mathrm{~s}, 3 \mathrm{H}), 2.73$ (brd, $J=13.7 \mathrm{~Hz}, 1 \mathrm{H}), 2.59(\mathrm{~m}, 2 \mathrm{H}), 2.45(\mathrm{dd}, J=18.8,11.4 \mathrm{~Hz}, 1 \mathrm{H}), 2.19(\mathrm{~m}, 1 \mathrm{H}), 2.05(\mathrm{~m}$, $2 \mathrm{H}), 1.82(\mathrm{~s}, 3 \mathrm{H}), 1.65(\mathrm{~s}, 6 \mathrm{H}), 0.96(\mathrm{~d}, J=6.5 \mathrm{~Hz}, 3 \mathrm{H}) \mathrm{ppm} ;{ }^{13} \mathrm{C} \mathrm{NMR}\left(150 \mathrm{MHz}, \mathrm{CDCl}_{3}\right)$ : $\delta=195.8,194.6,170.3,165.5,156.5,137.3,132.8,132.3,131.6$ (2 C), 131.3 (2 C), 131.0, 130.0, $129.3,127.9,127.1,122.5,120.3,120.2,111.5,66.2,64.7,55.8,51.2,31.4,30.9,30.0,29.5$, 25.9, 25.5, 17.9, 14.9 ppm; HRMS (MALDI): calcd for $\mathrm{C}_{33} \mathrm{H}_{35} \mathrm{O}_{6} \mathrm{Br}\left[\mathrm{MH}^{+}\right]$: 607.1690, found: 607.1680 .

54 (1:1 mixture of $\Delta^{8,9}$ geometrical isomers): $R_{\mathrm{f}}=0.40$ (silica gel, hexane/EtOAc 2:1); IR (film) $v_{\max }=2958,1772,1720,1686,1642,1589,1511,1458,1376,1301,1268,1174,1115,1069$,

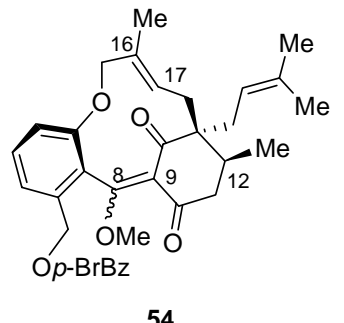

54

$1035,1010,987,929,850,758 \mathrm{~cm}^{-1} ;{ }^{1} \mathrm{H}$ NMR $\left(600 \mathrm{MHz}, \mathrm{CDCl}_{3}\right): \delta=7.94$ $(\mathrm{d}, J=7.7 \mathrm{~Hz}, 2 \mathrm{H}), 7.93(\mathrm{~d}, J=7.7 \mathrm{~Hz}, 2 \mathrm{H}), 7.58(\mathrm{~d}, J=7.7 \mathrm{~Hz}, 2 \mathrm{H})$, $7.57(\mathrm{~d}, J=8.7 \mathrm{~Hz}, 2 \mathrm{H}), 7.42(\mathrm{t}, J=7.7 \mathrm{~Hz}, 1 \mathrm{H}), 7.41(\mathrm{t}, J=7.7 \mathrm{~Hz}, 1 \mathrm{H})$, $7.26(\mathrm{~d}, J=7.7 \mathrm{~Hz}, 1 \mathrm{H}), 7.25(\mathrm{~d}, J=7.7 \mathrm{~Hz}, 1 \mathrm{H}), 7.12(\mathrm{~d}, J=8.7 \mathrm{~Hz}, 1$ H), $7.06(\mathrm{~d}, J=7.7 \mathrm{~Hz}, 1 \mathrm{H}), 5.49(\mathrm{~d}, J=13.1 \mathrm{~Hz}, 1 \mathrm{H}), 5.48(\mathrm{~d}, J=13.2$ Hz, $1 \mathrm{H}$ ), 5.42 (brt, $J=7.6 \mathrm{~Hz}, 1 \mathrm{H}), 5.29$ (d, $J=13.2 \mathrm{~Hz}, 1 \mathrm{H}$ ), 5.25 (d, $J=13.1 \mathrm{~Hz}, 1 \mathrm{H}), 5.19$ $(\mathrm{dd}, J=8.8,5.5 \mathrm{~Hz}, 1 \mathrm{H}), 5.05$ (brt, $J=7.1 \mathrm{~Hz}, 1 \mathrm{H}), 4.88$ (brd, $J=8.8 \mathrm{~Hz}, 1 \mathrm{H}), 4.61(\mathrm{~d}, J=$ $11.0 \mathrm{~Hz}, 1 \mathrm{H}), 4.55(\mathrm{~d}, J=11.0 \mathrm{~Hz}, 1 \mathrm{H}), 4.27(\mathrm{~d}, J=11.0 \mathrm{~Hz}, 1 \mathrm{H}), 4.07(\mathrm{~d}, J=11.0 \mathrm{~Hz}, 1 \mathrm{H})$, 3.57 (s, 3 H), 3.55 (s, 3 H), 2.81 (dd, $J=17.6,4.4$ Hz, 1 H), $2.65-2.50(\mathrm{~m}, 5 \mathrm{H}), 2.35$ - 2.20 (m, 5 H), $2.10(\mathrm{~m}, 2 \mathrm{H}), 1.74(\mathrm{dd}, J=14.2,5.4 \mathrm{~Hz}, 1 \mathrm{H}), 1.67$ (s, $3 \mathrm{H}), 1.63(\mathrm{~s}, 3 \mathrm{H}), 1.61(\mathrm{~s}, 3 \mathrm{H})$, $1.57(\mathrm{~s}, 6 \mathrm{H}), 1.51(\mathrm{~s}, 3 \mathrm{H}), 0.98(\mathrm{~d}, J=6.6 \mathrm{~Hz}, 3 \mathrm{H}), 0.94(\mathrm{~d}, J=6.6 \mathrm{~Hz}, 3 \mathrm{H}) \mathrm{ppm} ;{ }^{13} \mathrm{C}$ NMR $\left(150 \mathrm{MHz}, \mathrm{CDCl}_{3}\right): \delta=201.7,199.2,195.8,195.4,174.9,173.9,165.5,165.4,155.2,155.0$, 
139.1, 139.0, 134.7, 134.1, 133.9, 132.8, 131.8 (2 C), 131.7 (2 C), 131.5, 131.3 (4 C), 128.8, $128.4,128.3,128.2$, 127.6, 125.9, 125.4, 123.4, 123.1, 122.6, 121.4, 120.9, 120.7, 120.5, 119.8, 82.4, 80.1, 65.9, 65.6, 57.8, 57.6, 54.7, 54.4, 45.5, 45.3, 34.7, 32.6, 31.5, 30.8, 30.8, 29.5, 26.1, 26.0, 22.6, 18.0, 17.8, 17.5, 16.5, 16.0, 15.1, 14.1 ppm; HRMS (MALDI): calcd for $\mathrm{C}_{33} \mathrm{H}_{35} \mathrm{O}_{6} \mathrm{Br}$ $[\mathrm{MNa}]^{+}: 629.1509$, found: 629.1513 .

55: $R_{\mathrm{f}}=0.55$ (silica gel, hexane/EtOAc 2:1); IR (film) $v_{\max }=2956,2931,1721,1671,1634$, 1584, 1466, 1450, 1318, 1267, 1116, 1103, 1011, 976, 848, 757, $735 \mathrm{~cm}^{-1}$; ${ }^{1} \mathrm{H}$ NMR (500 MHz,

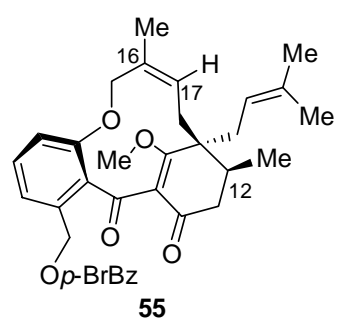

$\left.\mathrm{CDCl}_{3}\right): \delta=8.01(\mathrm{~d}, J=9.1 \mathrm{~Hz}, 2 \mathrm{H}), 7.58(\mathrm{~d}, J=8.2 \mathrm{~Hz}, 2 \mathrm{H}), 7.38(\mathrm{t}, J=$ $8.2 \mathrm{~Hz}, 1 \mathrm{H}), 7.20(\mathrm{~d}, J=7.4 \mathrm{~Hz}, 1 \mathrm{H}), 7.01(\mathrm{~d}, J=8.2 \mathrm{~Hz}, 1 \mathrm{H}), 5.81(\mathrm{~d}, J$ = $14.6 \mathrm{~Hz}, 1 \mathrm{H}), 5.80(\mathrm{~m}, 1 \mathrm{H}), 5.57$ (d, $J=14.6 \mathrm{~Hz}, 1 \mathrm{H}), 4.94$ (brs, $1 \mathrm{H})$, $4.36(\mathrm{AB}$ system, $\Delta \delta=0.0263 \mathrm{ppm}, J=9.9 \mathrm{~Hz}, 2 \mathrm{H}), 3.83(\mathrm{~s}, 3 \mathrm{H}), 2.82$ (brd, $J=11.9 \mathrm{~Hz}, 1 \mathrm{H}), 2.54(\mathrm{t}, J=11.9 \mathrm{~Hz}, 1 \mathrm{H}), 2.40(\mathrm{~m}, 1 \mathrm{H}), 2.35-$ $2.22(\mathrm{~m}, 2 \mathrm{H}), 2.14(\mathrm{dd}, J=14.6,9.1 \mathrm{~Hz}, 1 \mathrm{H}), 2.05(\mathrm{dd}, J=11.9,5.5 \mathrm{~Hz}, 1 \mathrm{H}), 1.95(\mathrm{~s}, 3 \mathrm{H})$, $1.71(\mathrm{~s}, 3 \mathrm{H}), 1.66(\mathrm{~s}, 3 \mathrm{H}), 0.92(\mathrm{~d}, J=6.5 \mathrm{~Hz}, 3 \mathrm{H}) \mathrm{ppm} ;{ }^{13} \mathrm{C} \mathrm{NMR}\left(125 \mathrm{MHz}, \mathrm{CDCl}_{3}\right): \delta=$ 198.5, 197.5, 177.1, 165.5, 156.8, 138.6, 133.7, 132.6, 132.0, 131.6 (2 C), 131.4 (2 C), 131.3, 129.4, 128.2, 127.9, 122.1, 121.0, 119.8, 114.9, 68.2, 64.6, 60.6, 49.3, 41.6, 32.7, 32.3, 30.1, 26.0, 24.1, 18.0, $15.1 \mathrm{ppm}$; HRMS (MALDI): calcd for $\mathrm{C}_{33} \mathrm{H}_{35} \mathrm{O}_{6} \mathrm{Br}[\mathrm{MNa}]^{+}: 629.1509$, found: 629.1527.

\section{Introduction of $\Delta^{11,12}$ double bond in macrocycles 53 and 54 (Scheme 11):}

To a solution of macrocycle 53 or $54(70 \mathrm{mg}, 0.115 \mathrm{mmol})$ in anhydrous THF $(4 \mathrm{~mL})$ at $-78^{\circ} \mathrm{C}$ was added slowly a solution of LiHMDS (150 $\mu \mathrm{L}, 1 \mathrm{M}$ in THF). The resulting solution was allowed to warm to $-10^{\circ} \mathrm{C}\left(25^{\circ} \mathrm{C}\right.$ for 54) over a period of $1 \mathrm{~h}$. After re-cooling to $-78^{\circ} \mathrm{C}\left(-60^{\circ} \mathrm{C}\right.$ for 54) a solution of $\mathrm{PhSeCl}(31 \mathrm{mg}, 0.16 \mathrm{mmol})$ in anhydrous THF (1 mL) was added slowly. The solution was allowed to warm to $0{ }^{\circ} \mathrm{C}\left(25^{\circ} \mathrm{C}\right.$ for $\left.\mathbf{5 4}\right)$ over a period of $30 \mathrm{~min}$. At this stage, formation of the less polar intermediate selenide, was observed by TLC. Once this conversion was complete, saturated aq. $\mathrm{NH}_{4} \mathrm{Cl}(0.3 \mathrm{~mL})$ and $31 \%$ aq. $\mathrm{H}_{2} \mathrm{O}_{2}(0.3 \mathrm{ml})$ were added and the mixture was stirred for $1 \mathrm{~h}$ at $25^{\circ} \mathrm{C}$. The mixture was diluted with EtOAc $(10 \mathrm{~mL})$ and the layers were separated. The organic phase was washed with $\mathrm{H}_{2} \mathrm{O}(5 \mathrm{~mL})$ and brine $(5 \mathrm{~mL})$ and then dried $\left(\mathrm{MgSO}_{4}\right)$. The solvent was removed in vacuo to afford, after purification by flash column 
chromatography (silica gel, EtOAc:hexane 1:6 $\rightarrow$ 1:3), $56(43 \mathrm{mg}, 61 \%)$ as a syrup, or 57 (37 $\mathrm{mg}, 51 \%)$ as a syrup accompanied by recovered $54(10.5 \mathrm{mg}, 15 \%)$.

56: $R_{\mathrm{f}}=0.33$ (silica gel, hexane/EtOAc 2:1); IR (film) $v_{\max }=2971,2914,1717,1650,1619$, 1590, 1532, 1464, 1397, 1376, 1326, 1269, 1116, 1104, 1011, 986, 757, $734 \mathrm{~cm}^{-1}$; ${ }^{1} \mathrm{H}$ NMR $(500$

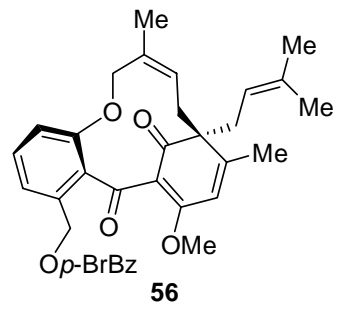

$\left.\mathrm{MHz}, \mathrm{CD}_{3} \mathrm{CN}\right): \delta=7.88(\mathrm{~d}, J=9.1 \mathrm{~Hz}, 2 \mathrm{H}), 7.59(\mathrm{~d}, J=8.3 \mathrm{~Hz}, 2 \mathrm{H}), 7.38$

$(\mathrm{t}, J=8.3 \mathrm{~Hz}, 1 \mathrm{H}), 7.17(\mathrm{~d}, J=7.4 \mathrm{~Hz}, 1 \mathrm{H}), 7.01(\mathrm{~d}, J=8.3 \mathrm{~Hz}, 1 \mathrm{H}), 6.50$ (brs, $1 \mathrm{H}), 5.50(\mathrm{~d}, J=13.2 \mathrm{~Hz}, 1 \mathrm{H}), 5.38$ (brt, $J=8.3 \mathrm{~Hz}, 1 \mathrm{H}), 5.30$ (d, $J$ $=13.2 \mathrm{~Hz}, 1 \mathrm{H}), 4.44(\mathrm{~d}, J=9.2 \mathrm{~Hz}, 1 \mathrm{H}), 4.43$ (brt, $J=6.4 \mathrm{~Hz}, 1 \mathrm{H}), 4.11$ (brd, $J=9.2 \mathrm{~Hz}, 1 \mathrm{H}), 3.85$ (s, $3 \mathrm{H}), 2.81(\mathrm{brd}, J=13.7 \mathrm{~Hz}, 1 \mathrm{H}), 2.50$ (dd, $J=12.8,10.1 \mathrm{~Hz}, 1 \mathrm{H}), 2.32(\mathrm{~m}, 2 \mathrm{H}), 2.02(\mathrm{~d}, J=1.8 \mathrm{~Hz}, 3 \mathrm{H}), 1.81(\mathrm{~s}, 3 \mathrm{H}), 1.56$ (s, $3 \mathrm{H}), 1.47$ (s, $3 \mathrm{H}$ ) ppm; ${ }^{13} \mathrm{C}$ NMR (125 MHz, $\left.\mathrm{CDCl}_{3}\right): \delta=200.5$ (br), 192.0, 171.5 (br), 165.5, 159.5, $157.4,136.6,135.0,133.5,131.5$ (2 C), 131.3 (2 C), 131.2, 130.8, 129.3, 127.6, 125.5, 123.1 (br), 119.0 (br), 114.9 (br), 107.8, 106.9 (br), 69.5 (br), 65.3, 58.6, 57.9, 53.4, 39.9, 25.5, 23.3 (br), 20.3, 18.0 ppm; HRMS (MALDI): calcd for $\mathrm{C}_{33} \mathrm{H}_{33} \mathrm{O}_{6} \mathrm{Br}$ [MNa] $]^{+}$627.1353, found: 627.1362 .

57: (mixture of $\Delta^{8,9}$ geometrical isomers in a 3:1 ratio): $R_{\mathrm{f}}=0.29$ (silica gel, hexane/EtOAc 2:1); IR (film) $v_{\max }=2918,1722,1650,1621,1533,1454,1267,1101,1011,758,735 \mathrm{~cm}^{-1} ;{ }^{1} \mathrm{H}$ NMR

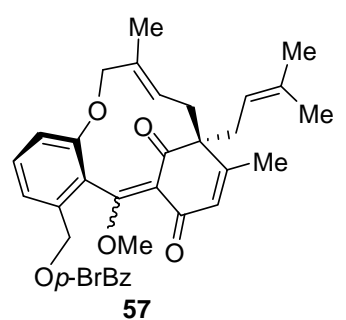

57

(600 MHz, $\mathrm{CDCl}_{3}$, major (E)-isomer): $\delta=7.95(\mathrm{~d}, J=9.1 \mathrm{~Hz}, 2 \mathrm{H}), 7.59$ $(\mathrm{d}, J=8.3 \mathrm{~Hz}, 2 \mathrm{H}), 7.38(\mathrm{t}, J=7.9 \mathrm{~Hz}, 1 \mathrm{H}), 7.23(\mathrm{~d}, J=7.9 \mathrm{~Hz}, 1 \mathrm{H}), 7.17$ $(\mathrm{d}, J=8.3 \mathrm{~Hz}, 1 \mathrm{H}), 6.30(\mathrm{~d}, J=1.3 \mathrm{~Hz}, 1 \mathrm{H}), 5.56(\mathrm{brd}, J=11.4 \mathrm{~Hz}, 1 \mathrm{H})$, $5.54(\mathrm{~d}, J=13.2 \mathrm{~Hz}, 1 \mathrm{H}), 5.29(\mathrm{~d}, J=13.2 \mathrm{~Hz}, 1 \mathrm{H}), 4.62$ (brt, $J=6.5 \mathrm{~Hz}$, $1 \mathrm{H}), 4.50(\mathrm{~d}, J=11.9 \mathrm{~Hz}, 1 \mathrm{H}), 4.43(\mathrm{~d}, J=11.9 \mathrm{~Hz}, 1 \mathrm{H}), 3.51(\mathrm{~s}, 3 \mathrm{H})$, $2.86(\mathrm{dd}, J=14.5,6.6 \mathrm{~Hz}, 1 \mathrm{H}), 2.31(\mathrm{~m}, 2 \mathrm{H}), 2.16(\mathrm{dd}, J=14.5,7.1 \mathrm{~Hz}, 1 \mathrm{H}), 1.95(\mathrm{~s}, 3 \mathrm{H})$, $1.57(\mathrm{~s}, 3 \mathrm{H}), 1.54(\mathrm{~s}, 3 \mathrm{H}), 1.35(\mathrm{~s}, 3 \mathrm{H}) \mathrm{ppm} ;{ }^{13} \mathrm{C} \mathrm{NMR}\left(125 \mathrm{MHz}, \mathrm{CDCl}_{3}\right.$, major $(E)$-isomer): $\delta=199.2,185.6,173.1,165.5,155.8,154.0,139.0,135.3,134.3,131.8$ (2 C), 131.3 (2 C), 130.6, 129.2, 128.8, 128.3, 125.0, 122.4, 120.2, 119.8, 119.6, 118.6, 76.4, 65.4, 57.3, 57.1, 37.5, 34.5, 25.8, 19.8, 19.1, 18.0 ppm; HRMS (MALDI): calcd for $\mathrm{C}_{33} \mathrm{H}_{33} \mathrm{O}_{6} \mathrm{Br}[\mathrm{MNa}]^{+}:$627.1353, found: 627.1361.

One-pot basic hydrolysis of the $p$-bromobenzoate and vinylogous methyl ester moieties of 56 and 57 (Scheme 11): 
To a solution of 56 or $57(35 \mathrm{mg}, 0.058 \mathrm{mmol})$ at $25^{\circ} \mathrm{C}$ in absolute $\mathrm{MeOH}(4 \mathrm{~mL})$ was added finely powdered $\mathrm{K}_{2} \mathrm{CO}_{3}(24 \mathrm{mg}, 0.174 \mathrm{mmol}$ ). The mixture was stirred for $11 \mathrm{~h}$ (40 min for 57) at the same temperature. Hydrolysis of the $p$-bromobenzoate and formation of the intermediate more polar benzylic alcohol was observed by TLC. In order to facilitate the hydrolysis of the vinylogous methyl ester moiety, five drops of $\mathrm{H}_{2} \mathrm{O}$ were added and the mixture was stirred for a further $3 \mathrm{~h}(30 \mathrm{~min}$ for 57$)$ at $25^{\circ} \mathrm{C}$. The reaction mixture was then diluted with $\mathrm{H}_{2} \mathrm{O}(6 \mathrm{~mL})$ and washed twice with hexane/Et $2 \mathrm{O}(3: 1,5 \mathrm{~mL})$ to remove organic impurities. The aqueous phase was acidified to $\mathrm{pH}=1$ with $1 \mathrm{M} \mathrm{HCl}$ and saturated with solid $\mathrm{NaCl}$. The suspension was then extracted twice with EtOAc $(5 \mathrm{~mL})$. The organic phase was washed with brine $(5 \mathrm{~mL})$, dried $\left(\mathrm{MgSO}_{4}\right)$ and concentrated in vacuo to afford benzylic alcohol 58 (21 mg, 90\%) as a yellow oil or benzylic alcohol $59(23 \mathrm{mg}, 96 \%)$ as an amorphous solid. The $(Z)$ and $(E)$ configuration at $\Delta^{16,17}$ double bond in $\mathbf{5 8}$ and $\mathbf{5 9}$, respectively, were unambiguously confirmed by extensive NOE studies (see Scheme 11).

58: $R_{\mathrm{f}}=0.44$ (silica gel, hexane/EtOAc, 1:1); IR (film): $v_{\max }=3436,2920,1651,1549,1449$, 1261, 1008, 914, $726 \mathrm{~cm}^{-1} ;{ }^{1} \mathrm{H}$ NMR $\left(600 \mathrm{MHz}, \mathrm{CDCl}_{3}\right): \delta=15.71(\mathrm{~s}, 1 \mathrm{H}), 7.38(\mathrm{t}, J=7.7 \mathrm{~Hz}$,

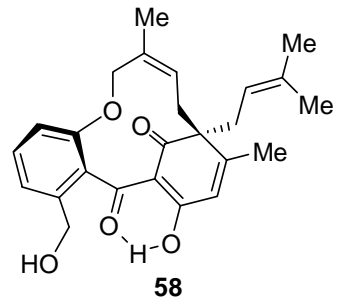

${ }^{13} \mathrm{C}$ NMR $\left(150 \mathrm{MHz}, \mathrm{CDCl}_{3}\right): \delta=198.8,181.0,164.1,156.4,139.8,136.0,134.4,131.9,131.7$, 131.4, 124.1, 123.6, 122.2, 117.8, 115.1, 113.1, 69.7, 64.2, 59.2, 39.0, 37.1, 25.7, 23.2, 20.1, 18.1; HRMS (MALDI) calcd for $\mathrm{C}_{25} \mathrm{H}_{27} \mathrm{O}_{4}\left[\mathrm{MH}-\mathrm{H}_{2} \mathrm{O}\right]^{+}$: 391.1904; found: 391.1905. MS (ESI) $409\left(\mathrm{MH}^{+}\right), 431\left(\mathrm{MNa}^{+}\right)$.

59: $R_{\mathrm{f}}=0.32$ (silica gel, hexane/EtOAc, 1:1); IR (film): $v_{\max }=3412,2920,1651,1556,1455$, 1262, 997, 866, $736 \mathrm{~cm}^{-1} ;{ }^{1} \mathrm{H}$ NMR (500 MHz, $\left.\mathrm{CDCl}_{3}\right): \delta=16.54$ (s, $\left.1 \mathrm{H}\right), 7.29$ (t, $J=8.2 \mathrm{~Hz}, 1$

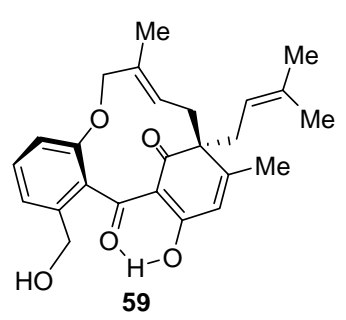

H), $7.12(\mathrm{~d}, J=8.2 \mathrm{~Hz}, 1 \mathrm{H}), 7.09$ (d, $J=7.4 \mathrm{~Hz}, 1 \mathrm{H}), 6.38$ (s, $1 \mathrm{H}), 5.41$ (br d, $J=12.0 \mathrm{~Hz}, 1 \mathrm{H}), 4.66(\mathrm{~d}, J=12.3 \mathrm{~Hz}, 1 \mathrm{H}), 4.56(\mathrm{~d}, J=11.4 \mathrm{~Hz}, 1$ H), 4.55 (br dd, $J=7.4,5.5 \mathrm{~Hz}, 1 \mathrm{H}), 4.49$ (d, $J=12.3 \mathrm{~Hz}, 1 \mathrm{H}), 4.31$ (d, $J$ $=11.4 \mathrm{~Hz}, 1 \mathrm{H}), 2.99(\mathrm{dd}, J=14.2,6.8 \mathrm{~Hz}, 1 \mathrm{H}), 2.44(\mathrm{t}, J=12.8 \mathrm{~Hz}, 1 \mathrm{H})$, 
2.33 (br d, $J=13.8 \mathrm{~Hz}, 1 \mathrm{H}), 2.25$ (dd, $J=14.6,6.4 \mathrm{~Hz}, 1 \mathrm{H}), 2.11$ (s, $3 \mathrm{H}), 1.58$ (br s, $3 \mathrm{H}), 1.57$ (br s, $3 \mathrm{H}), 1.33$ (s, $3 \mathrm{H}) ;{ }^{13} \mathrm{C}$ NMR $\left(125 \mathrm{MHz}, \mathrm{CDCl}_{3}\right): \delta=200.0,198.6,183.0,164.9$, 153.5, 139.6, 135.7, 134.9, 132.2, 129.6, 128.1, 123.3, 122.9, 118.3, 118.0, 114.2, 76.3, 63.7, 58.8, 38.3, 34.4, 25.7, 20.1 19.7, 18.0; HRMS (MALDI) calcd for $\mathrm{C}_{25} \mathrm{H}_{27} \mathrm{O}_{4}\left[\mathrm{MH}-\mathrm{H}_{2} \mathrm{O}\right]^{+}$: 391.1904 ; found: 391.1898. MS (ESI) $409\left(\mathrm{MH}^{+}\right), 431\left(\mathrm{MNa}^{+}\right)$.

\section{Completion of the total synthesis of coleophomones B and C (Scheme 11):}

To a solution of $\mathrm{CrO}_{3}(29 \mathrm{mg}, 0.29 \mathrm{mmol})$ in $\mathrm{CH}_{2} \mathrm{Cl}_{2}(2 \mathrm{~mL})$ at $0{ }^{\circ} \mathrm{C}$ was added pyridine $(47 \mathrm{mg}$, $0.58 \mathrm{mmol}$ ). The solution was allowed to warm to $25^{\circ} \mathrm{C}$ and was stirred for 20 min prior to the addition of benzylic alcohol $58(20 \mathrm{mg}, 0.049 \mathrm{mmol})$ at the same temperature. Complete consumption of the starting material after $2 \mathrm{~h}$ was observed by TLC. The solvent was removed in vacuo and the residue was taken up in $\mathrm{Et}_{2} \mathrm{O}(2 \mathrm{~mL})$ and filtered through a short pad of celite. Once again, the solvent was removed in vacuo. The residue was taken up in $1 \mathrm{M}$ aq. $\mathrm{K}_{2} \mathrm{CO}_{3}(5$ $\mathrm{mL})$ and washed with hexane/ $\mathrm{Et}_{2} \mathrm{O}(7: 3,5 \mathrm{~mL})$ to remove organic impurities. The aqueous phase was carefully acidified with $1 \mathrm{M} \mathrm{HCl}$ to $\mathrm{pH}=1$ and then extracted twice with EtOAc $(5 \mathrm{~mL})$. The layers were separated and the organic phase dried $\left(\mathrm{MgSO}_{4}\right)$ and concentrated in vacuo to afford coleophomone C (16 mg, 81\%) as a yellow amorphous solid.

3 (coleophomone C): $R_{\mathrm{f}}=0.42$ (silica gel, hexane/EtOAc 2:1); IR (film) $v_{\max }=2920,1713$, 1693, 1649, 1555, 1454, 1262, 1029, $790 \mathrm{~cm}^{-1} ;{ }^{1} \mathrm{H}$ NMR $\left(500 \mathrm{MHz}, \mathrm{CDCl}_{3}\right): \delta=16.02(\mathrm{~s}, 1 \mathrm{H})$,

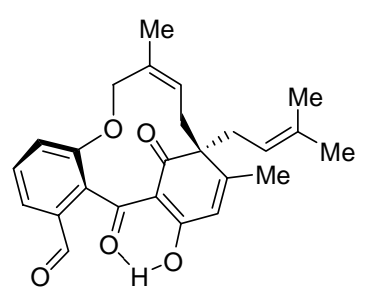

3: coleophomone $\mathrm{C}$ 10.02 (s, $1 \mathrm{H}), 7.67$ (d, J = 7.4 Hz, $1 \mathrm{H}), 7.53$ (dd, J = 8.3, 7.4 Hz, $1 \mathrm{H})$, $7.20(\mathrm{~d}, J=8.3 \mathrm{~Hz}, 1 \mathrm{H}), 6.34$ (brs, $1 \mathrm{H}), 5.28$ (m, $1 \mathrm{H}), 4.52$ (brt, $J=6.4$ $\mathrm{Hz}, 1 \mathrm{H}), 4.40(\mathrm{~d}, J=8.3 \mathrm{~Hz}, 1 \mathrm{H}), 4.14(\mathrm{~d}, J=8.3 \mathrm{~Hz}, 1 \mathrm{H}), 2.84(\mathrm{dd}, J=$ 13.7, 5.0 Hz, 1 H), 2.68 (dd, $J=13.7,11.0 \mathrm{~Hz}, 1 \mathrm{H}), 2.23$ (m, $2 \mathrm{H}), 2.10$ (brs, $3 \mathrm{H}), 1.83$ (brs, $3 \mathrm{H}), 1.57$ (brs, $3 \mathrm{H}$ ), 1.51 (brs, $3 \mathrm{H}$ ) ppm; ${ }^{13} \mathrm{C}$ NMR $\left(150 \mathrm{MHz} \mathrm{CDCl}_{3}\right): \delta=198.5,196.7,190.9,181.7,164.7,156.1,135.7,134.6,134.5,134.2$, 130.7, 123.9, 123.4, 122.3, 121.9, 117.8, 113.1, 70.6, 59.2, 39.1, 36.8, 25.7, 22.9, 20.2, 18.1 ppm; HRMS (MALDI): calcd for $\mathrm{C}_{25} \mathrm{H}_{26} \mathrm{O}_{5}[\mathrm{MNa}]^{+}: 429.1672$, found: 429.1671 .

A solution of benzylic alcohol $59(20 \mathrm{mg}, 0.049 \mathrm{mmol})$ in $\mathrm{Et}_{2} \mathrm{O}(3 \mathrm{~mL})$ was treated with $\mathrm{MnO}_{2}$ ( $85 \mathrm{mg}, 0.98 \mathrm{mmol}$ ) and the resulting mixture was heated to reflux for $8 \mathrm{~h}$. Filtration through a short pad of celite was followed by removal of the solvent in vacuo to afford coleophomone B (14.5 mg, 73\%) as a yellow amorphous solid. 


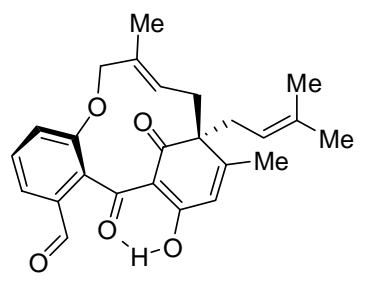

2: coleophomone B

2 (coleophomone B): $R_{\mathrm{f}}=0.40$ (silica gel, hexane/EtOAc 1:1); IR (film)

$v_{\max }=2926,2855,1701,1660,1558,1457,1261,1113,912,866,822$, 736, $703 \mathrm{~cm}^{-1} ;{ }^{1} \mathrm{H}$ NMR (600 MHz, $\left.\mathrm{CDCl}_{3}\right): \delta=16.41(\mathrm{~s}, 1 \mathrm{H}), 9.95$ (s, 1 H), $7.59(\mathrm{~d}, J=7.7 \mathrm{~Hz}, 1 \mathrm{H}), 7.48(\mathrm{t}, J=7.7 \mathrm{~Hz}, 1 \mathrm{H}), 7.44(\mathrm{~d}, J=7.7$ $\mathrm{Hz}, 1 \mathrm{H}), 6.36$ (brs, $1 \mathrm{H}), 5.43$ (brd, $J=11.0 \mathrm{~Hz}, 1 \mathrm{H}), 4.56$ (d, $J=12.0$ $\mathrm{Hz}, 1 \mathrm{H}), 4.56$ (brm, $1 \mathrm{H}), 4.40$ (d, $J=12.0 \mathrm{~Hz}, 1 \mathrm{H}), 2.91$ (dd, $J=15.2,3.3 \mathrm{~Hz}, 1 \mathrm{H}), 2.37$ (dd, $J$ $=13.2,12.1 \mathrm{~Hz}, 1 \mathrm{H}), 2.28(\mathrm{dd}, J=13.2,3.3 \mathrm{~Hz}, 1 \mathrm{H}), 2.25$ (dd, $J=15.3,7.7 \mathrm{~Hz}, 1 \mathrm{H}), 2.08$ (brs, $3 \mathrm{H}), 1.59$ (brs, $3 \mathrm{H}$ ), 1.54 (brs, $3 \mathrm{H}$ ), 1.32 (brs, $3 \mathrm{H}$ ) ppm; ${ }^{13} \mathrm{C} \mathrm{NMR}\left(150 \mathrm{MHz}, \mathrm{CDCl}_{3}\right): \delta=$ 197.9, 196.9, 190.4, 182.2, 164.7, 153.8, 135.9, 135.0, 134.4, 133.4, 129.5, 129.1, 126.0, 125.0, 122.5, 118.5, 113.3, 76.8, 58.5, 38.4, 34.3, 25.7, 20.2, 19.8, 18.1 ppm; HRMS (MALDI): calcd for $\mathrm{C}_{25} \mathrm{H}_{26} \mathrm{O}_{5}[\mathrm{MNa}]^{+}: 429.1672$, found: 429.1673 .

\section{Abortive attempts to synthesize coleophomone A. Preparation of spirocycle 62 (Scheme 12):}

To a solution of $\mathrm{CrO}_{3}(26 \mathrm{mg}, 0.26 \mathrm{mmol})$ in $\mathrm{CH}_{2} \mathrm{Cl}_{2}(2 \mathrm{~mL})$ at $0{ }^{\circ} \mathrm{C}$ was added pyridine (42 $\mathrm{mg}$, $0.52 \mathrm{mmol}$ ). The solution was allowed to warm to $25^{\circ} \mathrm{C}$ and stirred for $20 \mathrm{~min}$. The solution was

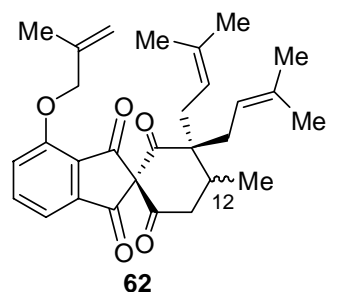

re-cooled to $0{ }^{\circ} \mathrm{C}$, prior to the addition of benzaldehyde $\leftrightarrow$ spirocycle $\mathbf{6 0}$ (17 $\mathrm{mg}, 0.037 \mathrm{mmol})$. The starting material was shown to have been completely consumed after 5 min by TLC. The solvent was removed in vacuo and the residue was taken up in $\mathrm{Et}_{2} \mathrm{O}(2 \mathrm{~mL})$ and filtered through a short pad of celite. The organic solution was dried $\left(\mathrm{MgSO}_{4}\right)$ and concentrated in vacuo to afford a crude residue that was purified by flash column chromatography (silica gel, EtOAc:hexane 1:2) to furnish pure $62(7 \mathrm{mg}, 39 \%)$ as a pale yellow oil.

62 (mixture of stereoisomers at $\mathrm{C} 12$ in a 2.5:1 ratio); $R_{\mathrm{f}}=0.45$ (silica gel, hexane/EtOAc $3: 1$ ); ${ }^{1} \mathrm{H}$ $\operatorname{NMR}\left(500 \mathrm{MHz}, \mathrm{CDCl}_{3}\right): \delta=7.75$ (t, $J=8.2 \mathrm{~Hz}, 1 \mathrm{H}$, minor), 7.71 (t, $J=8.2 \mathrm{~Hz}, 1 \mathrm{H}$, major), 7.59 (d, $J=7.3 \mathrm{~Hz}, 1 \mathrm{H}$, minor), 7.55 (d, $J=7.3 \mathrm{~Hz}, 1 \mathrm{H}$, major), 7.24 (d, $J=8.2 \mathrm{~Hz}, 1 \mathrm{H}$, major), 7.22 (d, $J=8.2 \mathrm{~Hz}, 1 \mathrm{H}$, minor), 5.21 (brs, $1 \mathrm{H}$, major), 5.20 (brs, $1 \mathrm{H}$, minor), 5.05 (brs, $1 \mathrm{H}$, major plus $1 \mathrm{H}$, minor), 5.03 (brt, $J=6.4 \mathrm{~Hz}, 1 \mathrm{H}$, major plus $1 \mathrm{H}$, minor), 4.88 (brt, $J=6.4$ $\mathrm{Hz}, 1 \mathrm{H}$, minor), 4.85 (brt, $J=6.4 \mathrm{~Hz}, 1 \mathrm{H}$, major), 4.64 (s, $2 \mathrm{H}$, major), 4.62 (s, $2 \mathrm{H}$, minor), $2.96(\mathrm{~m}, 1 \mathrm{H}$, major plus $1 \mathrm{H}$, minor), $2.76(\mathrm{~m}, 1 \mathrm{H}$, minor), $2.64(\mathrm{~m}, 3 \mathrm{H}$, major plus $2 \mathrm{H}$, minor), 2.45 (m, $2 \mathrm{H}$, major plus $2 \mathrm{H}$, minor), 2.25 (dd, $J=14.6,8.2 \mathrm{~Hz}, 1 \mathrm{H}$, major plus $1 \mathrm{H}$, 
minor), 1.86 (brs, $3 \mathrm{H}$, major), 1.85 (brs, $3 \mathrm{H}$, minor), 1.70 (brs, $3 \mathrm{H}$, major plus $3 \mathrm{H}$, minor), 1.67 (brs, $3 \mathrm{H}$, major), 1.66 (brs, $3 \mathrm{H}$, minor), 1.62 (brs, $3 \mathrm{H}$, major plus $3 \mathrm{H}$, minor), 1.61 (brs, 3 $\mathrm{H}$, major plus $3 \mathrm{H}$, minor), 1.12 (d, $J=6.4 \mathrm{~Hz}, 3 \mathrm{H}$, major), 1.09 (d, $J=6.4 \mathrm{~Hz}, 3 \mathrm{H}$, minor) ppm; ${ }^{13} \mathrm{C}$ NMR (125 MHz, $\mathrm{CDCl}_{3}$, major): $\delta=204.7,202.3,190.8,189.9,157.2,145.5,139.0$, 137.4, 134.7, 134.2, 119.4, 118.7, 118.1, 116.4, 115.7, 113.6, 85.1, 72.6, 55.1, 43.7, 30.3, 30.1, 29.7, 28.6, 26.1, 26.0, 19.2, 17.9, 15.7 ppm. HRMS (MALDI): calcd for $\mathrm{C}_{29} \mathrm{H}_{34} \mathrm{O}_{5}[\mathrm{MNa}]^{+}$: 485.2298, found: 485.2303.

\section{Olefin metathesis of 67a, 68a, and 69a. Preparation of spirocycles 70, 72, and 74 (Scheme} 13):

To a degassed solution of regioisomeric vinylogous esters $67 \mathbf{a}$ or $68 \mathbf{a}$ or $69 \mathbf{a}(50 \mathrm{mg}, 0.078$ mmol, bubbling for 15 min with $\mathrm{Ar})$ in anhydrous $\mathrm{CH}_{2} \mathrm{Cl}_{2}(5 \mathrm{~mL})$ was added the second generation Grubbs catalyst $\mathbf{A}(13 \mathrm{mg}, 0.015 \mathrm{mmol})$ in one portion. The resulting pink solution was heated to reflux, with light excluded from the reaction vessel, until complete consumption of the starting material was observed by TLC ( $4 \mathrm{~h}$ for $67 \mathbf{a}, 8 \mathrm{~h}$ for $68 \mathrm{a}$ and $3 \mathrm{~h}$ for $69 \mathbf{a}$ ). The solvent was removed in vacuo and the residue was purified by flash column chromatography (silica gel, EtOAc:hexane $1: 9 \rightarrow 1: 2)$ to afford spirocycle 70 (30 mg, 67\%), an 1:5.6 inseparable mixture of macrocycle 71 and spirocycle 72 (34 mg, 75\% total yield), and a 1:2.4 inseparable mixture of macrocycle 73 and spirocycle 74 (32 $\mathrm{mg}, 71 \%$ total yield), respectively. When the mixture of 71 and 72, or the mixture of $\mathbf{7 3}$ and $\mathbf{7 4}$, were treated again with catalyst $\mathbf{A}$ for $48 \mathrm{~h}$, only spirocycles $72\left(1.1: 1\right.$ mixture of geometrical isomers at $\left.\Delta^{8,9}\right)$ and $\mathbf{7 4}$ were isolated as syrups in 50 and $41 \%$ overall yields, starting from 68a and 69a, respectively.

70: $R_{\mathrm{f}}=0.49$ (silica gel, hexane/EtOAc 2:1); IR (film) $v_{\max }=2918,1722,1660,1644,1589$, 1454, 1267, 1102, 1011, $757 \mathrm{~cm}^{-1} ;{ }^{1} \mathrm{H} \operatorname{NMR}\left(500 \mathrm{MHz}, \mathrm{CDCl}_{3}\right): \delta=7.95(\mathrm{~d}, J=9.1 \mathrm{~Hz}, 2 \mathrm{H})$,

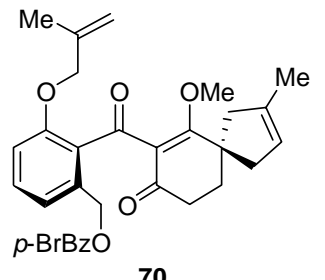
$7.55(\mathrm{~d}, J=9.1 \mathrm{~Hz}, 2 \mathrm{H}), 7.34(\mathrm{t}, J=8.2 \mathrm{~Hz}, 1 \mathrm{H}), 7.17(\mathrm{~d}, J=7.4 \mathrm{~Hz}, 1 \mathrm{H})$, $6.84(\mathrm{~d}, J=8.2 \mathrm{~Hz}, 1 \mathrm{H}), 5.55$ (s, $2 \mathrm{H}), 5.24$ (brs, $1 \mathrm{H}), 4.98$ (brs, $1 \mathrm{H}), 4.97$ (brs, $1 \mathrm{H}), 4.39$ (s, $2 \mathrm{H}), 3.83$ (s, $3 \mathrm{H}), 2.85$ (brd, $J=13.7 \mathrm{~Hz}, 2 \mathrm{H}), 2.30$ (m, $3 \mathrm{H}), 2.17(\mathrm{~d}, J=15.5 \mathrm{~Hz}, 1 \mathrm{H}), 1.85$ (m, $2 \mathrm{H}), 1.78$ (s, $3 \mathrm{H}), 1.74$ (brs, $3 \mathrm{H})$ ppm; ${ }^{13} \mathrm{C}$ NMR $\left(125 \mathrm{MHz}, \mathrm{CDCl}_{3}\right): \delta=197.0,195.7,183.2,165.5,156.4,140.2,137.8,137.4$, 131.8, 131.5 (2 C), 131.4 (2 C), 131.3, 129.7, 129.4, 127.8, 121.7, 121.2, 113.6, 111.7, 72.5, 
72.0, 65.1, 63.1, 48.8, 34.6, 33.7, 19.7, 19.3, $16.4 \mathrm{ppm}$; HRMS (MALDI): calcd for $\mathrm{C}_{31} \mathrm{H}_{31} \mathrm{O}_{6} \mathrm{Br}$ $[\mathrm{MNa}]^{+}:$601.1196, found: 601.1190.

74: $R_{\mathrm{f}}=0.23$ (silica gel, hexane/EtOAc 2:1); IR (film) $v_{\max }=2919,1719,1684,1647,1589$, $1467,1373,1267,1103,1070,1011,984,757 \mathrm{~cm}^{-1} ;{ }^{1} \mathrm{H}$ NMR $\left(500 \mathrm{MHz}, \mathrm{CDCl}_{3}\right): \delta=7.94(\mathrm{~d}, J$ $=9.1 \mathrm{~Hz}, 2 \mathrm{H}), 7.54(\mathrm{~d}, J=8.3 \mathrm{~Hz}, 2 \mathrm{H}), 7.28(\mathrm{dd}, J=8.3,7.3 \mathrm{~Hz}, 1 \mathrm{H})$,

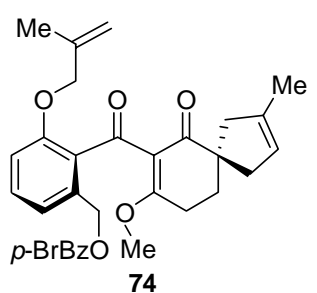
$7.10(\mathrm{~d}, J=7.3 \mathrm{~Hz}, 1 \mathrm{H}), 6.81(\mathrm{~d}, J=8.3 \mathrm{~Hz}, 1 \mathrm{H}), 5.48(\mathrm{~s}, 2 \mathrm{H}), 5.07$ (d, $J=$ $1.8 \mathrm{~Hz}, 1 \mathrm{H}), 4.99$ (brs, $1 \mathrm{H}), 4.95$ (brs, $1 \mathrm{H}), 4.35$ (s, $2 \mathrm{H}), 3.76$ (s, $3 \mathrm{H})$, $2.70-2.60$ (m, 4 H), 2.09 (brd, $J=13.7 \mathrm{~Hz}, 1 \mathrm{H}), 1.94$ (m, $3 \mathrm{H}), 1.77$ (s, 3 $\mathrm{H}), 1.63$ (brs, $3 \mathrm{H}) \mathrm{ppm} ;{ }^{13} \mathrm{C}$ NMR $\left(125 \mathrm{MHz}, \mathrm{CDCl}_{3}\right): \delta=198.4,195.7$, 175.5, 165.5, 156.0, 140.7, 137.2, 136.2, 131.5 (2 C), 131.4 (2 C), 130.8, 130.5, 129.4, 127.8, 121.3, 121.2, 121.1, 112.7, 111.9, 72.4, 64.9, 56.4, 50.3, 45.2, 41.1, 31.8, 23.5, 19.4, 16.3 ppm; HRMS (MALDI): calcd for $\mathrm{C}_{31} \mathrm{H}_{31} \mathrm{O}_{6} \mathrm{Br}[\mathrm{MNa}]^{+}$: 601.1196, found: 601.1184 .

\section{Total synthesis of coleophomone D (Scheme 14):}

To a solution of LDA at $-78{ }^{\circ} \mathrm{C}$, prepared by 20 min treatment of a solution of diisopropylamine $(0.554 \mathrm{~mL}, 4.22 \mathrm{mmol})$ in anhydrous THF $(10 \mathrm{~mL})$ with $n$-BuLi $(1.76 \mathrm{~mL}, 1.6 \mathrm{M}$ in hexane), was slowly added a solution of $75(0.39 \mathrm{~g}, 1.41 \mathrm{mmol})$ in THF:HMPA $(25: 1,10.4 \mathrm{~mL})$ and the resulting solution was slowly warmed to $0{ }^{\circ} \mathrm{C}$ over a $2 \mathrm{~h}$ period. The solution was re-cooled to $78^{\circ} \mathrm{C}$ and a solution of $\mathrm{PhSeCl}(0.404 \mathrm{~g}, 2.11 \mathrm{mmol})$ in anhydrous THF $(2 \mathrm{~mL})$ was added. The resulting solution was warmed to $20{ }^{\circ} \mathrm{C}$ over a period of $30 \mathrm{~min}$. At this stage, complete conversion of $\mathbf{7 5}$ to the intermediate selenide was observed by TLC. The solution was diluted with $\mathrm{Et}_{2} \mathrm{O}(20 \mathrm{~mL})$ and quenched with saturated aq. $\mathrm{NH}_{4} \mathrm{Cl}(10 \mathrm{~mL})$. The layers were separated and the organic phase was washed with brine $(10 \mathrm{~mL})$. The organic phase was then treated for 1 h at $45{ }^{\circ} \mathrm{C}$ with $31 \%$ aq. $\mathrm{H}_{2} \mathrm{O}_{2}(1.0 \mathrm{~mL})$. The organics were then washed with $\mathrm{H}_{2} \mathrm{O}(10 \mathrm{~mL})$ and brine $(10 \mathrm{~mL})$, dried $\left(\mathrm{MgSO}_{4}\right)$, and concentrated in vacuo to afford, after purification by flash column chromatography (silica gel, EtOAc:hexane 1:9), vinylogous ester $\mathbf{7 6}(0.30 \mathrm{~g}, 78 \%)$ as a syrup.

76: $R_{\mathrm{f}}=0.60$ (silica gel, hexane/EtOAc 2:1); IR (film) $v_{\max }=2971,2914,1658,1631,1582$, 1444, 1387, 1218, 1147, $848 \mathrm{~cm}^{-1} ;{ }^{1} \mathrm{H}$ NMR (500 MHz, $\left.\mathrm{CDCl}_{3}\right): \delta=6.00(\mathrm{brd}, J=2.2 \mathrm{~Hz}, 1 \mathrm{H})$,

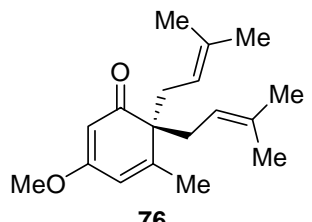

$5.41(\mathrm{~d}, J=2.2 \mathrm{~Hz}, 1 \mathrm{H}), 4.69$ (brt, $J=7.0 \mathrm{~Hz}, 2 \mathrm{H}), 3.72$ (s, $3 \mathrm{H}), 2.69$ (dd, $J$ 
$=14.3,7.0 \mathrm{~Hz}, 2 \mathrm{H}), 2.26(\mathrm{dd}, J=14.6,7.0 \mathrm{~Hz}, 2 \mathrm{H}), 1.90(\mathrm{~d}, J=1.1 \mathrm{~Hz}, 3 \mathrm{H}), 1.57$ (brs, $12 \mathrm{H})$;

${ }^{13} \mathrm{C}$ NMR $\left(125 \mathrm{MHz}, \mathrm{CDCl}_{3}\right): \delta=204.2,171.7,155.3,133.6,120.6,118.5,99.8,57.2,55.4$, 38.0, 25.7, 19.1, 18.0 ppm. HRMS (MALDI): calcd for $\mathrm{C}_{18} \mathrm{H}_{26} \mathrm{O}_{2}[\mathrm{MH}]^{+}$: 275.2005, found: 275.2006 .

To a solution of vinylogous ester 76, prepared above, $(0.20 \mathrm{~g}, 0.73 \mathrm{mmol})$ in $\mathrm{MeOH}: \mathrm{H}_{2} \mathrm{O}(2: 1,9$ $\mathrm{mL})$ was added $\mathrm{LiOH} \bullet \mathrm{H}_{2} \mathrm{O}(0.153 \mathrm{~g}, 3.65 \mathrm{mmol})$. The resulting mixture was heated for $12 \mathrm{~h}$ at $80{ }^{\circ} \mathrm{C}$. The solution was cooled to room temperature, neutralized with $1 \mathrm{M} \mathrm{HCl}$, diluted with $\mathrm{H}_{2} \mathrm{O}$ $(10 \mathrm{~mL})$, and extracted twice with EtOAc $(10 \mathrm{~mL})$. The layers were separated and the organic phase was washed with brine, dried $\left(\mathrm{MgSO}_{4}\right)$, concentrated in vacuo and purified by flash column chromatography (silica gel, EtOAc:hexane 1:2) to afford 1,3-cylohexedione 77 (0.173 g, $91 \%)$ as a syrup.

77: $R_{\mathrm{f}}=0.12$ (silica gel, hexane/EtOAc 2:1); IR (film) $v_{\max }=2916,2618,1650,1556,1445$, $1385,1313,1222,877,734,614 \mathrm{~cm}^{-1} ;{ }^{1} \mathrm{H}$ NMR $\left(600 \mathrm{MHz}, \mathrm{CDCl}_{3}\right.$, keto:enol $\left.=1.4: 1\right): \delta=6.26$ (s, $1 \mathrm{H}$, keto), $6.10(\mathrm{brs}, 1 \mathrm{H}, \mathrm{enol}), 5.70(\mathrm{~d}, J=1.7 \mathrm{~Hz}, 1 \mathrm{H}$, enol), 4.77 (brt, $J$
77 $1.90(\mathrm{~d}, J=1.3 \mathrm{~Hz}, 3 \mathrm{H}$, enol), 1.62 (s, $6 \mathrm{H}$, keto), 1.58 (s, $6 \mathrm{H}$, keto plus $6 \mathrm{H}$, enol) 1.57 (s, $6 \mathrm{H}$, enol); ${ }^{13} \mathrm{C}$ NMR $\left(150 \mathrm{MHz}, \mathrm{CDCl}_{3}\right): \delta=207.1,193.8,186.9,185.6,158.8,135.8,134.0(2 \mathrm{C})$, 129.8, 127.3, 119.7, 119.6, 117.8, (2 C), 105.5, 58.1, 53.7, 52.5, 36.3 (2 C), 35.4, 25.8, 25.6 (2 C), 19.8, 18.6, 18.0 (2 C), 17.8 ppm. HRMS (MALDI): calcd for $\mathrm{C}_{17} \mathrm{H}_{24} \mathrm{O}_{2}[\mathrm{MNa}]^{+}$: 283.1668, found: 283.1665 .

To a solution of aldehyde $\mathbf{7 9}^{38}(0.73 \mathrm{~g}, 3.50 \mathrm{mmol})$ in anhydrous toluene $(25 \mathrm{~mL})$ at $0{ }^{\circ} \mathrm{C}$ was added slowly a solution of $\mathrm{Et}_{2} \mathrm{AlCN}(3.5 \mathrm{~mL}, 1 \mathrm{M}$ in toluene) and the resulting solution was stirred for $2 \mathrm{~h}$ at the same temperature. The mixture was then diluted with EtOAc $(30 \mathrm{~mL})$ and treated with saturated aq. sodium potassium tartrate $(30 \mathrm{~mL})$ for 30 min with vigorous stirring. The layers were separated and the organic phase was dried $\left(\mathrm{MgSO}_{4}\right)$, concentrated in vacuo, and purified with flash chromatography (silica gel, EtOAc:hexane 1:4 $\rightarrow 1: 2$ ) to afford the desired cyanohydrin $81(0.485 \mathrm{~g}, 59 \%)$ as a syrup, accompanied by the regioisomeric migrated acetate $80(0.123 \mathrm{~g}, 15 \%)$. 
81: $R_{\mathrm{f}}=0.18($ silica gel, hexane/EtOAc $2: 1) ;{ }^{1} \mathrm{H}$ NMR $\left(500 \mathrm{MHz}, \mathrm{CDCl}_{3}\right): \delta=7.40(\mathrm{dd}, J=8.4$, $7.7 \mathrm{~Hz}, 1 \mathrm{H}), 7.29$ (brd, $J=7.7 \mathrm{~Hz}, 1 \mathrm{H}), 6.98$ (brd, $J=8.4 \mathrm{~Hz}, 1 \mathrm{H}), 5.85$ (d, $J=5.9 \mathrm{~Hz}, 1 \mathrm{H})$, $5.36(\mathrm{~d}, J=12.3 \mathrm{~Hz}, 1 \mathrm{H}), 5.28(\mathrm{~d}, J=12.3 \mathrm{~Hz}, 1 \mathrm{H}), 4.49$ (d, $J=5.9 \mathrm{~Hz}, 1 \mathrm{H}), 3.86(\mathrm{~s}, 3 \mathrm{H})$, 2.05 (s, $3 \mathrm{H}$ ) ppm; ${ }^{13} \mathrm{C}$ NMR (125 MHz, $\left.\mathrm{CDCl}_{3}\right): \delta=172.2,159.2,136.9,131.2,122.1,120.3$,

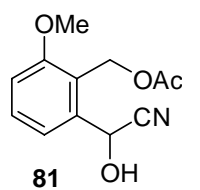
119.5, 112.8, 61.4, 57.8, 56.4, 21.4 ppm. HRMS (MALDI): calcd for $\mathrm{C}_{12} \mathrm{H}_{13} \mathrm{O}_{4} \mathrm{~N}$ $[\mathrm{MNa}]^{+}: 258.0737$, found: 258.0737 .

A solution of cyanohydrin 81, prepared above, $(0.44 \mathrm{~g}, 1.87 \mathrm{mmol})$ in anhydrous $\mathrm{CH}_{2} \mathrm{Cl}_{2}(25 \mathrm{~mL})$ was heated to reflux for $4 \mathrm{~h}$ in the presence of PCC $(1.61 \mathrm{~g}, 7.48$ mmol). A sufficient amount of celite was then added to absorb the reaction mixture accompanied by vigorous stirring. The resulting paste was transferred directly on to the top of a silica gel column and subsequent flash column chromatography (EtOAc:hexane 1:3 $\rightarrow$ 1:2) afforded acyl cyanide $82(0.22 \mathrm{~g}, 51 \%)$ as white crystals.

82: M.p. $104-105^{\circ} \mathrm{C}\left(\mathrm{CDCl}_{3}\right) ; R_{\mathrm{f}}=0.33$ (silica gel, hexane/EtOAc 2:1); IR (film) $v_{\max }=1724$, 1672, 1588, 1472, 1281, 1247, 1013, $740 \mathrm{~cm}^{-1} ;{ }^{1} \mathrm{H}$ NMR (500 MHz, $\left.\mathrm{CDCl}_{3}\right): \delta=7.86(\mathrm{brd}, J=$

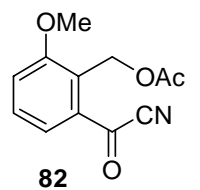

$7.7 \mathrm{~Hz}, 1 \mathrm{H}), 7.61(\mathrm{t}, J=8.1 \mathrm{~Hz}, 1 \mathrm{H}), 7.32(\mathrm{brd}, J=8.1 \mathrm{~Hz}, 1 \mathrm{H}), 5.45(\mathrm{~s}, 2 \mathrm{H}), 3.92$ $(\mathrm{s}, 3 \mathrm{H}), 2.03(\mathrm{~s}, 3 \mathrm{H}) \mathrm{ppm} ;{ }^{13} \mathrm{C} \mathrm{NMR}\left(125 \mathrm{MHz}, \mathrm{CDCl}_{3}\right): \delta=170.7,168.0,159.0$, 133.5, 130.6, 126.3, 125.4, 118.4, 113.3, 56.5, 56.4, 20.7 ppm. HRMS (MALDI): calcd for $\mathrm{C}_{12} \mathrm{H}_{11} \mathrm{O}_{4} \mathrm{~N}[\mathrm{MNa}]^{+}: 256.0580$, found: 256.0582 .

To a solution of acyl cyanide $82(0.12 \mathrm{~g}, 0.52 \mathrm{mmol})$ in THF (7 mL) was added 1,3cyclohexedione $77(0.135 \mathrm{~g}, 0.52 \mathrm{mmol})$ followed by $\mathrm{Et}_{3} \mathrm{~N}(72 \mu \mathrm{L}, 0.52 \mathrm{mmol})$. The resulting solution was stirred for $72 \mathrm{~h}$ at $25^{\circ} \mathrm{C}$. The solution was diluted with $\mathrm{Et}_{2} \mathrm{O}(10 \mathrm{~mL})$, washed with $1 \mathrm{M} \mathrm{HCl}(5 \mathrm{~mL})$ and brine $(10 \mathrm{~mL})$ and then dried $\left(\mathrm{MgSO}_{4}\right)$. The solvent was removed in vacuo and the residue was purified by flash column chromatography (silica gel, EtOAc:hexane 1:4 $\rightarrow$ $1: 3)$ to provide the pure coupled product $(0.193 \mathrm{~g}, 80 \%)$ as a yellow syrup. ${ }^{1} \mathrm{H}$ NMR spectrum of this tricarbonyl compound revealed a complicated mixture of enol and keto tautomers.

The coupled product $(0.193 \mathrm{~g}, 0.41 \mathrm{mmol})$, prepared above, was dissolved in $\mathrm{MeOH}(15 \mathrm{~mL})$ and was stirred with finely powdered $\mathrm{K}_{2} \mathrm{CO}_{3}(0.172 \mathrm{~g}, 1.25 \mathrm{mmol})$ for $24 \mathrm{~h}$ at $25^{\circ} \mathrm{C}$. The mixture was diluted with $\mathrm{H}_{2} \mathrm{O}(20 \mathrm{~mL})$ and the aqueous phase was washed twice with $10 \mathrm{~mL}$ hexane/ $\mathrm{Et}_{2} \mathrm{O}(7: 3)$ to remove organic impurities. The aqueous phase was then acidified to $\mathrm{pH}=1$ with $1 \mathrm{M} \mathrm{HCl}$, saturated with solid $\mathrm{NaCl}$, and extracted twice with EtOAc (15 mL). Finally, the 
organic phase was washed with brine, dried $\left(\mathrm{MgSO}_{4}\right)$ and concentrated in vacuo to afford benzylic alcohol $83(0.165 \mathrm{~g}, 94 \%)$ as a yellow syrup.

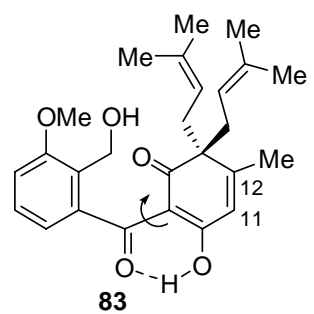

83 (mixture of two keto atropisomers in 3:2 ratio in $\mathrm{CDCl}_{3}$ ): $R_{\mathrm{f}}=0.31$ (silica gel, hexane:EtOAc, 2:1); IR (film): $v_{\max }=2967,2922,1776,1732,1651$, 1614, 1589, 1484, 1270, 1079, $728 \mathrm{~cm}^{-1} ;{ }^{1} \mathrm{H} \mathrm{NMR}\left(600 \mathrm{MHz}, \mathrm{CDCl}_{3}\right): \delta=$ 8.47 (d, $J=7.7 \mathrm{~Hz}, 1 \mathrm{H}$, minor), 8.07 (d, $J=7.7 \mathrm{~Hz}, 1 \mathrm{H}$, major), 7.41 (t, $J=$ $7.7 \mathrm{~Hz}, 1 \mathrm{H}$, minor), $7.35(\mathrm{t}, J=7.7 \mathrm{~Hz}, 1 \mathrm{H}$, major), $7.04(\mathrm{~d}, J=7.7 \mathrm{~Hz}, 1$ H, minor), 7.02 (d, $J=7.7 \mathrm{~Hz}, 1 \mathrm{H}$, major), 6.29 (s, $1 \mathrm{H}$, minor), 6.27 (s, $1 \mathrm{H}$, major), 5.61 (s, 2 H, major), 5.58 (s, $2 \mathrm{H}$, minor), 4.85 (br t, $J=6.6 \mathrm{~Hz}, 2 \mathrm{H}$, major), 4.82 (br t, $J=6.6 \mathrm{~Hz}, 2 \mathrm{H}$, minor), 3.90 (s, $3 \mathrm{H}$, minor), 3.90 (s, $3 \mathrm{H}$, major), 2.83 (m, $2 \mathrm{H}$, minor and $2 \mathrm{H}$, major), 2.32 (dd, $J=14.3,6.6 \mathrm{~Hz}, 2 \mathrm{H}$, major), 2.24 (dd, $J=14.3,6.6 \mathrm{~Hz}, 2 \mathrm{H}$, minor), 1.97 (s, $3 \mathrm{H}$, minor), 1.95 (s, $3 \mathrm{H}$, major), 1.55 (s, $6 \mathrm{H}$, major and $6 \mathrm{H}$, minor), 1.49 (s, $6 \mathrm{H}$, major and $6 \mathrm{H}$, minor); ${ }^{13} \mathrm{C}$ NMR (150 MHz, $\mathrm{CDCl}_{3}$ ): $\delta=202.2$ (minor), 200.3 (major), 186.7 (minor), 185.6 (major), 177.2 (major), 176.9 (minor), 157.3 (minor), 156.0 (major), 153.4 (2C), 134.3, 134.2, 134.1, 133.8, 131.1, 130.5, 130.1, 129.8, 121.5, 120.5, 118.9 (2C), 118.8 (2C), 114.7 (minor), 114.6 (major), 113.9 (minor), 113.8 (major), 75.8 (major), 75.5 (minor), 57.5 (minor), 56.9 (major), 55.5 (major and minor), 36.5 (2 C minor), 36.3 (2 C major), 25.7 (2 C major and $2 \mathrm{C}$ minor), 19.5 (minor), 19.2 (major), 18.1 (major), 17.9 (minor); HRMS (MALDI) calcd for $\mathrm{C}_{26} \mathrm{H}_{30} \mathrm{O}_{4} \mathrm{Na}\left[\mathrm{MNa}-\mathrm{H}_{2} \mathrm{O}\right]^{+}$: 429.2042; found: 429.2097; MS (ESI) $407\left(\mathrm{MH}^{+}-\mathrm{H}_{2} \mathrm{O}\right), 429\left(\mathrm{MNa}^{+}-\mathrm{H}_{2} \mathrm{O}\right)$.

A solution of benzylic alcohol $83(0.15 \mathrm{~g}, 0.35 \mathrm{mmol})$ in $\mathrm{Et}_{2} \mathrm{O}(15 \mathrm{~mL})$ was treated with $\mathrm{MnO}_{2}$ $(0.308 \mathrm{~g}, 3.50 \mathrm{mmol})$ and the resulting mixture was refluxed for $4 \mathrm{~h}$. Filtration through a short pad of celite followed by solvent evaporation in vaсио afforded coleophomone D (0.122 g, 83\%) as a yellow amorphous solid. Coleophomone D exists as a complex and dynamic mixture of isomers (see Scheme 1). The spectral data we obtained for the synthetically derived sample is in full accord with the data reported ${ }^{3}$ for the naturally occurring sample.

Reference numbers used in this experimental section refer to the same reference numbers used in the main body of the manuscript. 